\title{
Message self and social relevance increases intentions to share content: \\ Correlational and causal evidence from six studies
}

\begin{abstract}
Danielle Cosme ${ }^{1}$, Christin Scholz ${ }^{2}$, Hang-Yee Chan ${ }^{2}$, Bruce P. Doré ${ }^{3}$, Prateekshit Pandey, José
Carreras-Tartak $^{1}$, Nicole Cooper ${ }^{1}$, Alexandra Paul ${ }^{1}$, Shannon M. Burns ${ }^{1}$, and Emily B. Falk ${ }^{1,4,5,6}$
\end{abstract}

\author{
${ }^{1}$ University of Pennsylvania, Annenberg School for Communication \\ ${ }^{2}$ University of Amsterdam, Amsterdam School of Communication Research \\ ${ }^{3}$ McGill University, Desautels Faculty of Management \\ ${ }^{4}$ University of Pennsylvania, Wharton Marketing Department \\ ${ }^{5}$ University of Pennsylvania, Department of Psychology \\ ${ }^{6}$ University of Pennsylvania, Wharton Operations, Information and Decisions Department
}

Word count: 6,355

Data, code, and preregistration links:

https://osf.io/bgs5y

https://github.com/cnlab/self-social-sharing

Address correspondence to:

Danielle Cosme, danielle.cosme@asc.upenn.edu

Emily Falk, emily.falk@asc.upenn.edu

Funding: This research was funded in part by a Small Business Innovation Research grant in collaboration with Charles River Analytics and CACI through the Defense Advanced Research Project Agency (DARPA) under contract number 140D0419C0093, and the Army Research Office and was accomplished under grant number W911NF-18-1-0244. The views and conclusions contained in this document are those of the authors and should not be interpreted as representing the official policies, either expressed or implied, of the Army Research Office or the U.S. Government. The U.S. Government is authorized to reproduce and distribute reprints for Government purposes notwithstanding any copyright notation herein. 


\begin{abstract}
Information sharing within social networks can catalyze widespread attitudinal and behavioral change and the chance to share information with others has been characterized as inherently valuable to people. But what are the sources of value and how might they be leveraged to promote sharing? We test ideas from the value-based virality model that the value of sharing increases when people perceive messages as more relevant to themselves and to people they know, resulting in stronger intentions to share. We extend this work by considering how sharing context — broadcasting to a wide audience or narrowcasting directly to someone - may alter these relationships. Six online studies with adults in the United States (N participants $=3,727$; messages $=362$; message ratings $=30,954)$ showed robust evidence that self and social relevance are positively and uniquely related to sharing intentions within- and between-person. Specification curve analysis showed these relationships were consistent across message content (COVID-19, voting, general health, climate change), medium (social media post and news articles), and sharing context (broad- and narrowcasting). A preregistered experiment showed that manipulating the self and social relevance of messages through a framing manipulation causally increased sharing intentions. These causal effects were mediated by changes in both self and social relevance, but the relative strength of the causal pathways differed depending on sharing context. These findings extend existing models of information sharing, and highlight self and social relevance as psychological mechanisms that motivate information sharing that can be targeted to promote sharing across contexts.
\end{abstract}

Keywords: sharing, social media, self-relevance, norms, influence, virality 
At this moment, we are facing various global crises - from the COVID-19 pandemic, to climate change - that require large scale attitudinal and behavioral change. For widespread action, it is vital that accurate and persuasive information is transmitted between people. When people share information with one another, it also affects what people perceive as normative (Tankard \& Paluck, 2016) and hence how they behave (Cialdini et al., 1991; Jeong \& Bae, 2017). Sharing information is a fundamental aspect of human social interaction that catalyzes social change (Barberá et al., 2015) and has been characterized as inherently valuable to people (Tamir \& Mitchell, 2012; Vijayakumar et al., 2020). In the current study, we examine potential sources of this value and how they can be leveraged to promote information sharing. Bringing together insights from psychology, neuroscience, communication, and marketing, we test the hypotheses that when people see information as being relevant to themselves and to people in their social networks, these sources of value motivate them to share with others. Further, we add nuance to prior theorizing by examining how the sharing context affects the psychological processes that are most important in deciding what to share.

\section{Sharing as a value-based decision}

The value-based virality model asserts that decisions to share information are a particular case of value-based decision making which involves selecting choice options based on their relative value (Falk \& Scholz, 2018; Scholz et al., 2017). The perceived costs and benefits of each option are implicitly and explicitly weighed and integrated into a common currencysubjective value - that enables comparison between options (Levy \& Glimcher, 2012). Neuroscientific research demonstrates that decisions to share information (Baek et al., 2017; Scholz, Jovanova, et al., 2020) and population-level outcomes such as popularity, campaign effectiveness, and message virality (Doré et al., 2019; Falk et al., 2012; Genevsky \& Knutson, 
SHARING, SELF AND SOCIAL RELEVANCE

2015), are associated with increased activity in the brain's valuation system. But what factors might be integrated during subjective value calculation when deciding whether or not to share information?

Research on persuasion and communication highlights characteristics of the information and of the individual sharer as important features (Kümpel et al. 2015 and Capella et al., 2015). For example, how positive (Al-Rawi, 2017), emotionally arousing (Berger \& Milkman, 2012), controversial (Kim, 2020), or useful (Kim, 2015) content is perceived to be, is positively associated with sharing and virality. An individual's implicit motives, such as social bonding (Baek et al., 2019) and impression management (Ihm \& Kim, 2018), and explicit goals, such as information seeking (Lee \& Ma, 2012) and persuasion (Berger, 2014), also shape sharing behavior. Applying the value-based decision framework to these findings suggest a parsimonious mechanism (i.e., valuation) through which a sharer can account for, compare, and integrate these disparate factors when deciding to share.

From a translational perspective, any of these factors might be used as levers to increase or decrease the subjective value associated with the decision to share. However, these diverse inputs can be broadly characterized as self-related or social concerns, which in turn can be captured by self and social relevance - the perceived relevance to other people within the person's social network. For example, self-relevance encompasses various self-related processes and motivations, such as emotional experience, goals, self-expression, self-enhancement. This paper focuses on these two sources of value because they are central in psychological theories of persuasion and social influence (for a review, see Falk \& Scholz, 2018) and represent practical targets for sharer-focused interventions to promote sharing behavior. Such interventions could frame information in different ways to enhance its perceived self or social relevance, thereby 
SHARING, SELF AND SOCIAL RELEVANCE

increasing subjective value and the likelihood of sharing, without altering the content itself (which may distract or distort the information). Because this approach relies on framing alone and could operate through any number of idiosyncratic factors an individual views as self or socially relevant (e.g., the content is viewed as useful, in line with one's goals, or affords the opportunity to connect), it may alter subjective value in a relatively scalable way.

\section{Self and social relevance}

Information related to the self is expected to have higher subjective value for several reasons. First, there are well-documented egocentric biases in which individuals tend to pay greater attention to (Humphreys \& Sui, 2015), process information more efficiently (Markus, 1977; Meyer \& Lieberman, 2018), and over-value objects and attributes perceived as being related to the self (Beer \& Hughes, 2010; Kahneman et al., 1991; Mezulis et al., 2004; Taylor \& Brown, 1988). Second, there is substantial overlap between brain regions supporting selfreferential processing and valuation (Berkman et al., 2017; Chavez et al., 2017; D’Argembeau, 2013; Pfeifer \& Berkman, 2018), suggesting that these processes are intimately intertwined. Finally, disclosing information about oneself is thought to be intrinsically rewarding and therefore subjectively valued (Tamir \& Mitchell, 2012).

Social relevance is also hypothesized to increase subjective value in the context of sharing decisions. Humans have a fundamental need to belong (Baumeister \& Leary, 1995) and can connect by sharing information. Sharing feels good and is associated with activity in the brain's reward and valuation systems (Tamir et al., 2015). When communicating, individuals consider what is relevant to their audience in order to tailor their communication effectively (Berger, 2014; Echterhoff et al., 2009) and this ability is supported by the tendency to spontaneously consider and predict others' mental states (Koster-Hale \& Saxe, 2013; Mildner \& 
Tamir, 2021; Thornton et al., 2018). In addition, individuals are motivated to conform to social norms and are therefore likely to consider what people will think of them if they share (Schultz et al., 2007).

Integrating this evidence with the observations from neuroscientific research on valuebased decision making, the value-based virality model (Scholz et al., 2017) proposes that information that is perceived as more self and/or socially relevant will have higher subjective value during valuation, and therefore be more likely to be shared and go viral. There is indirect evidence from neuroimaging studies supporting this hypothesis; brain regions associated with self-referential processing and social cognition are related to sharing intentions (Baek et al., 2017; Scholz, Baek, et al., 2020) and population-level virality (Scholz et al., 2017). However, it is unclear whether: 1) explicit evaluations of self and social relevance-which are less costly to measure - are similarly related to sharing behavior, 2) self and social relevance can be causally manipulated to promote information sharing, and 3) these relationships generalize across contexts, such as the type of content being shared and the audience being targeted.

\section{Broad- versus narrowcast sharing}

While much of the existing psychological work on sharing has treated it as a single, homogeneous behavior, some prior work in communication and marketing has highlighted the need for nuance, distinguishing different forms of sharing based on the audience size (Barasch \& Berger, 2014). "Broadcasting" is sharing information with a large and often ill-defined group of individuals (e.g., via public posts on social media), whereas "narrowcasting" is sharing information with one or a small group of well-defined individuals (e.g., via a direct message). Audience size is an important contextual variable for sharing research, because it has implications for the reach and impact of the shared piece of information, as well as for the impact 
sharing may have on the information sharer (Scholz, Baek et al., 2020). For instance, narrowcasting leads to fewer additional exposures to the shared content than broadcasting, but since the shared information is specifically targeting one or few individuals, narrowcasting may be more likely to elicit prolonged attention and responses. Further, broadcasters compared to narrowcasters generally deal with greater potential diversity and uncertainty regarding the opinions and existing knowledge of their audiences, which may have implications for the sharing of controversial content or the way in which content is shared. In fact, research has shown that information sharers engage in so-called audience tuning, meaning that they adjust what they share and how they do so, depending on audience characteristics (Echterhoff et al., 2009), and the most tuning will occur when tailoring messages to a specific person when narrowcasting. Pronounced differences in the social and content-related implications of narrow- and broadcasting bring with them related differences in the psychological motivations and experiences of broad- and narrowcasters.

Importantly, self-related and social motivations have been hypothesized to play differential roles in broad- versus narrowcasting. For instance, some have argued that self-related motivations, such as self-presentation, self-enhancement, and self-expression (Berger, 2014), play a larger role in broadcasting because uncertainty about the precise make-up of the audience in terms of individual members, options, etc. leads sharers to focus more on themselves rather than specific audience characteristics. On the other hand, social motivations, such as connecting with or helping others (Berger, 2014), may be more important in narrowcasting because the audience is well-defined and messages are more easily tailored to meet the target's needs. Although initial research testing these hypotheses pitted self and other motivations against one another (Barasch \& Berger, 2014), neuroimaging research suggests that self and social 
processing are involved in both broad- and narrowcasting and that differences between sharing types are related to the relative degree of engagement (Scholz, Baek, et al., 2020). We extend prior work by integrating these findings with the value-based virality model, which did not originally consider contextual factors, such as audience size. Specifically, we test the hypothesis that contextual factors such as audience size change the relative importance of different decision attributes in terms of their impact on the overall computation of the value of sharing. That is, we expect that the relative contribution of self and social relevance to the calculation of subjective value varies based on the sharing audience — self-relevance would be expected to weigh more heavily during decisions to broadcast, and social relevance would be expected to weigh more heavily during decisions to narrowcast.

\section{The present research}

Across six online studies (participant $\mathrm{N}=3,727$; Table 1), we tested correlational relationships between the self and social relevance of informational messages and intentions to share them (Studies 1-6), and whether experimentally manipulating self and social relevance causally increases message sharing intentions (Study 6). We focused on messages about important societal issues (messages $\mathrm{N}=362$; message ratings $\mathrm{N}=30,954$ ), and assessed the generalizability of these relationships across message content (COVID-19, voting, general health, climate change) and medium (social media posts, newspaper articles). Given that self and social relevance may differentially contribute to decisions to share depending on the audience (Scholz, Baek, et al., 2020), we also examined how these relationships may differ as a function of sharing context (broadcast and narrowcast sharing). Because it is unclear whether the relationships between self and social relevance and sharing are driven by message-induced responses or by individual differences in the propensity to view content as self and/or socially 
relevant, we distinguished within- and between-person relationships using multilevel modeling. We also examined the robustness of these relationships across alternative model specifications and within specific subsets of the data using specification curve analysis.

Table 1

Overview of studies

\begin{tabular}{lccccc}
\hline Study & N & Content & Medium & Sharing type & Type \\
\hline Study 1 & 2081 & COVID-19 & Social media & Broadcast & Correlational \\
Study 2 & 547 & Voting & Social media & Broadcast & Correlational \\
Study 3 & 248 & Voting & Social media & Broad- \& narrowcast & Correlational \\
Study 4 & 139 & Health & Newspapers & Broadcast & Correlational \\
Study 5* & 315 & COVID-19 \& climate & Newspapers & Broad- \& narrowcast & Correlational \\
Study 6* & 397 & Health \& climate change & Newspapers & Broad- \& narrowcast Correlational \& causal \\
\hline \multicolumn{5}{l}{ Note. Study 1 combines data from four samples from the same project. *Preregistered study }
\end{tabular}

\section{Open practices statement}

Studies 1-4 used existing data, whereas Studies 5 and 6 were preregistered before collecting data (https://osf.io/bgs5y/registrations). The sample sizes for Studies 5 and 6 were based on power calculations described in the preregistrations. Standard operating procedures for Studies 5 and 6 are available online (https://osf.io/bgs5y/). The data and analysis code needed to reproduce the main analyses reported here are available online (https://github.com/cnlab/selfsocial-sharing). Individual demographic data is not posted publicly due to concerns related to potential identifiability of participants, but is available upon request. The messages used in this study are available online (https://osf.io/nfr7h/). In response to reviewer feedback noting the strong relationship between self and social relevance, we deviated from our preregistration by estimating mediation models that included self and social relevance as parallel mediators (versus only including one mediator as preregistered) to assess the specificity of the experimental effects on sharing intentions. For transparency, we also include the original, preregistered models in Supplementary Material. 


\section{Correlational analyses}

\section{Methods}

\section{Participants}

These analyses included data from six online studies $(\mathrm{N}=3,727)$ using convenience sampling. Participants were living in the United States and aged 18 to $81(M=38.1, S D=12.0)$. With respect to gender, participants identified as the following: $52.5 \%$ men, $46.6 \%$ women, $0.2 \%$ non-binary or third gender, $0.2 \%$ idenfitied as another category ("other"), and $0.4 \%$ preferred not to say. With respect to race and ethnicity (not reported in Study 4), participants identified as the following: $76.9 \%$ White, 11.5\% Hispanic or Latina/Latino/Latinx, 10.6\% Black or African American, 8.6\% Asian, 0.9\% More than one race, 0.8\% American Indian or Alaskan Native, $0.1 \%$ Native Hawaiian or Other Pacific Islander, $1.5 \%$ as another race (“other”), and $0.6 \%$ preferred not to say. Additional demographic information, demographic information by study, and the specific inclusion and exclusion criteria for each study is reported in Supplementary Material. Study 4 was conducted online through the Human Subjects Pool at the University of Pennsylvania; all other studies were conducted online through Amazon's Mechanical Turk (MTurk). All studies were approved by the University of Pennsylvania Institutional Review Board or deemed exempt from review, and all participants gave informed consent and were compensated financially or with course credit.

\section{Procedure}

Participants were exposed to 5-10 messages about COVID-19, voting, general health, or climate change (Table 1). In Studies 1-3, these messages were framed as social media posts, whereas in Studies 4-6 they were headlines and brief abstracts from New York Times newspaper articles. After reading each message, participants rated self-relevance (e.g., "This message is 
relevant to me") and social relevance (e.g., "This message is relevant to people I know"). Two types of sharing intentions were measured: broadcast and narrowcast. In all studies, participants rated their broadcast intention to share on social media (e.g., "I would share this article by posting on social media (on Facebook, Twitter, etc)"). In Studies 3, 5, and 6 they also rated their narrowcast intention to share directly with someone (e.g., "I would share this article directly with someone I know (via email, direct message, etc)"). The specific language and scales differed across studies; see Supplementary Material for study-specific details. Responses were z-scored within study in order to conduct analyses across studies.

\section{Statistical analyses}

We investigated the relationships between message self and social relevance and sharing intentions using multilevel modeling. We included self and social relevance in the same models to examine their unique relationships with sharing intentions (see Supplementary Material for separate models). Self and social relevance ratings were disaggregated into within and betweenperson variables. The within-person self and social relevance variables were level-1 predictors, centered within-person (i.e., "centered within context") and standardized across people within each study. These variables represent message-level deviations from a person's average self or social relevance rating. Each of the between-person variables were level-2 predictors created by averaging across the self or social relevance ratings of all messages to create a single average per person that was then grand-mean centered and standardized across people within each study. These variables represent person-level deviations from the average self or social relevance rating across people. All models were estimated using the lme4 (Version 1.1-26; Bates et al., 2015) and lmerTest (Version 3.1-3; Kuznetsova, Brockhoff, \& Chris-tensen, 2017) for significance testing in R (Version 3.6.3; R Core Team, 2020). Degrees of freedom ( $d f$ ) were calculated using the 
SHARING, SELF AND SOCIAL RELEVANCE

Satterthwaite approximation. All $p$-values reported are from two-tailed tests. The specification curve analysis was implemented using code adapted from specr (Masur \& Scharkow, 2020). All software packages used are listed in Supplementary Material.

Mega-analysis. We used a mega-analysis approach (Eisenhauer, 2021) to pool raw data from all six studies and precisely estimate the correlational relationships between self and social relevance, and sharing intentions, as a function of sharing type (broad- or narrowcasting). We estimated a single multilevel model with the within- and between-person self and social relevance variables, and their interactions with sharing type as predictors. We adopted the least constrained random effects structure that converged; intercepts and within-person self and social relevance were allowed to vary randomly across people and messages. Although the variance inflation factors (VIF) for the predictor variables were small to moderate (VIF range $=1.00$ 4.24), we conducted a sensitivity analysis to assess the impact of multicollinearity on the estimated regression coefficients. Specifically, we estimated the mega-analysis model in a subset of the data where message-level correlations (i.e., the correlation between self and social relevance for a given message) below $r=.70$. These analyses are presented in Supplementary Material; the results did not change appreciably from those reported in the main manuscript. For completeness, we also present analyses for each study individually as well as the mega-analysis estimated for self and social relevance separately (i.e., not in the same model) in Supplementary Material.

Specification curve analysis. We used specification curve analysis (SCA) to explore the robustness of the relationships between self and social relevance, and sharing intentions. Briefly, SCA can be used to map a collection of possible models that could be specified to test a given hypothesis (Simonsohn et al., 2020; Steegen et al., 2016). Because the studies in this manuscript 
varied with respect to content, medium, and sharing type, we used SCA to estimate the relationships between message self and social relevance, and sharing intentions within specific subsets of the data, as well as when adjusting for demographic covariates. Specifically, we included within- and between-person self and social relevance as predictors of interest and included each of the following demographic covariates: age, gender, race, ethnicity, highest degree completed, and household income. This resulted in a set of 7 possible model specifications for each relevance variable, including models with no demographic covariates. We then created 13 unique subsets of the data based on message content, medium, and sharing type (e.g., broadcasting across social media messages or narrowcasting across newspaper articles about COVID-19; see Supplementary Material for a full list of subsets), and estimated the set of model specifications for each relevance variable within each subset. Not all studies included the same demographic variables and therefore studies missing specific demographic covariates are not included in the estimation of the corresponding model specifications. Together, this resulted in 86 per relevance variable (a total of 344 model specifications). For each model specification, we extracted the standardized regression coefficient for the predictor of interest, ordered them by effect size, and plotted them to form a specification curve for each relevance variable separately. For each model specification in the curve, we visualized which relevance variable was the predictor of interest, the content type, medium, sharing type, and whether or not demographic covariates were included. In line with recent recommendations to avoid inflating the model space with poorly specified models (Giudice \& Gangestad, 2021), we conceptualize this set of analytic decisions as uncertain ("Type-U”) because the decision options are not clearly equivalent or nonequivalent, and treat these analyses as exploratory, focusing on descriptive rather than inferential statistics. 
SHARING, SELF AND SOCIAL RELEVANCE

\section{Results}

\section{Descriptives}

Table 2 shows the means, standard deviations, and correlations between the self and social-relevance survey ratings and sharing variables for each study separately. Within-person correlations were estimated using the rmcorr package (Bakdash \& Marusich, 2017).

Table 2

Means, standard deviations, and repeated measures correlations for each study

\begin{tabular}{|c|c|c|c|c|c|c|}
\hline Study & Variable & Range & $M(S D)$ & & $r[95 \% \mathrm{CI}]$ & \\
\hline & & & & self-relevance & social relevance & broadcast \\
\hline \multirow[t]{3}{*}{ Study 1} & Self & $1-7$ & $5.4(1.5)$ & - & - & - \\
\hline & Social & $1-7$ & $5.7(1.4)$ & $0.66[0.65,0.67]$ & - & - \\
\hline & Broadcast & $1-7$ & $4.5(2.0)$ & $0.45[0.43,0.47]$ & $0.45[0.43,0.46]$ & - \\
\hline \multirow[t]{3}{*}{ Study 2} & Self & $0-100$ & $63.0(30.7)$ & & & \\
\hline & Social & $0-100$ & $69.2(26.3)$ & $0.60[0.58,0.63]$ & & \\
\hline & Broadcast & $0-100$ & $49.2(35.9)$ & $0.31[0.27,0.34]$ & $0.31[0.27,0.35]$ & \\
\hline \multirow[t]{4}{*}{ Study 3} & Self & $0-100$ & $69.4(27.1)$ & - & - & - \\
\hline & Social & $0-100$ & 76.7 (21.7) & $0.69[0.65,0.72]$ & - & - \\
\hline & Broadcast & $0-100$ & $43.6(33.3)$ & $0.36[0.30,0.41]$ & $0.35[0.29,0.40]$ & - \\
\hline & Narrowcast & $0-100$ & $48.4(33.5)$ & $0.40[0.35,0.45]$ & $0.34[0.37,0.48]$ & $0.68[0.64,0.71]$ \\
\hline \multirow[t]{3}{*}{ Study 4} & Self & $0-10$ & $4.1(3.4)$ & - & - & - \\
\hline & Social & $0-10$ & $5.8(2.7)$ & $0.59[0.55,0.63]$ & - & - \\
\hline & Broadcast & $0-10$ & $3.8(3.4)$ & $0.64[0.61,0.68]$ & $0.55[0.50,0.59]$ & - \\
\hline \multirow[t]{4}{*}{ Study 5} & Self & $0-100$ & $56.8(29.8)$ & - & - & - \\
\hline & Social & $0-100$ & $61.5(27.9)$ & $0.71[0.70,0.73]$ & - & - \\
\hline & Broadcast & $0-100$ & $49.8(32.3)$ & $0.52[0.50,0.55]$ & $0.46[0.43,0.49]$ & - \\
\hline & Narrowcast & $0-100$ & $50.3(32.1)$ & $0.48[0.45,0.51]$ & $0.53[0.50,0.55]$ & $0.67[0.65,0.69]$ \\
\hline \multirow[t]{4}{*}{ Study 6} & Self & $0-100$ & $57.3(32.2)$ & - & - & - \\
\hline & Social & $0-100$ & $62.8(29.6)$ & $0.67[0.65,0.69]$ & - & - \\
\hline & Broadcast & $0-100$ & $47.2(34.6)$ & $0.49[0.46,0.51]$ & $0.47[0.44,0.49]$ & - \\
\hline & Narrowcast & $0-100$ & $48.8(33.5)$ & $0.48[0.45,0.50]$ & $0.57[0.55,0.60]$ & $0.59[0.57,0.61]$ \\
\hline
\end{tabular}

Note . Range $=$ scale range, broadcast $=$ broadcast sharing intentions, narrowcast $=$ narrowcast sharing intentions, self $=$ self-relevance, social $=$ social relevance .

\section{Mega-analysis}

With pooled data from all six studies, we estimated a single multilevel model to assess the relationship between within-person and between-person self and social relevance and 
intentions to share, and whether these relationships differ as a function of sharing type. Because the self and social relevance variables were included in the same model, the parameter estimates reflect their unique effects after adjusting for the other variables in the model. First, we report the main effects of these variables on broadcasting, which was the reference group for sharing type. Then, we report the interactions that test whether these relationships differed between broad- and narrowcast sharing intentions. Between-person relationships reflect average deviations from the group mean, whereas within-person relationships reflect deviations from a persons' mean.

Broadcasting. Integrating across studies revealed a moderate positive relationship with between-person self-relevance $(\beta=0.35,95 \% \mathrm{CI}[0.31,0.39])$ and a small positive relationship with between-person social relevance $(\beta=0.16,95 \% \mathrm{CI}[0.12,0.20])$. This indicates that people who tended to perceive messages as more self and socially relevant also tended to report higher sharing intentions. Within-person there were small positive relationships with self-relevance $(\beta=$ $0.18[0.17,0.20])$ and social relevance $(\beta=0.13,95 \%$ CI $[0.12,0.14])$, indicating that when people perceived messages as more self and socially relevant (compared to their own average perceived relevance), they also reported higher intentions to share it.

Broadcasting versus narrowcasting. Next we tested the interaction between each relevance variable and sharing type. Between people, the relationship between self-relevance and sharing intentions was weaker when narrowcasting compared to broadcasting $\left(\beta_{\text {interaction }}=-0.10\right.$, 95\% CI [-0.13, -0.07]), whereas the relationship between social relevance and sharing intentions was stronger when narrowcasting $\left(\beta_{\text {interaction }}=0.11,95 \% \mathrm{CI}[0.08,0.14]\right)$. This indicates that people who tend to rate messages as more relevant to themselves also tend to have higher sharing intentions when broadcasting compared to narrowcasting, whereas people who rate messages as more socially relevant have stronger sharing intentions when narrowcasting compared to 
broadcasting. The same pattern was observed for within-person self-relevance $\left(\beta_{\text {interaction }}=-0.06\right.$, $95 \%$ CI $[-0.08,-0.04])$ and social relevance $\left(\beta_{\text {interaction }}=0.11,95 \%\right.$ CI $\left.[0.09,0.13]\right)$. When people rated messages as more relevant to themselves, they had higher intentions to share them when broadcasting compared to narrowcasting, and when people rated messages as more socially relevant, they had higher intentions to share them when narrowcasting compared broadcasting. These relationships are visualized in Figure 1 and model parameters and statistics are presented in Table 3.

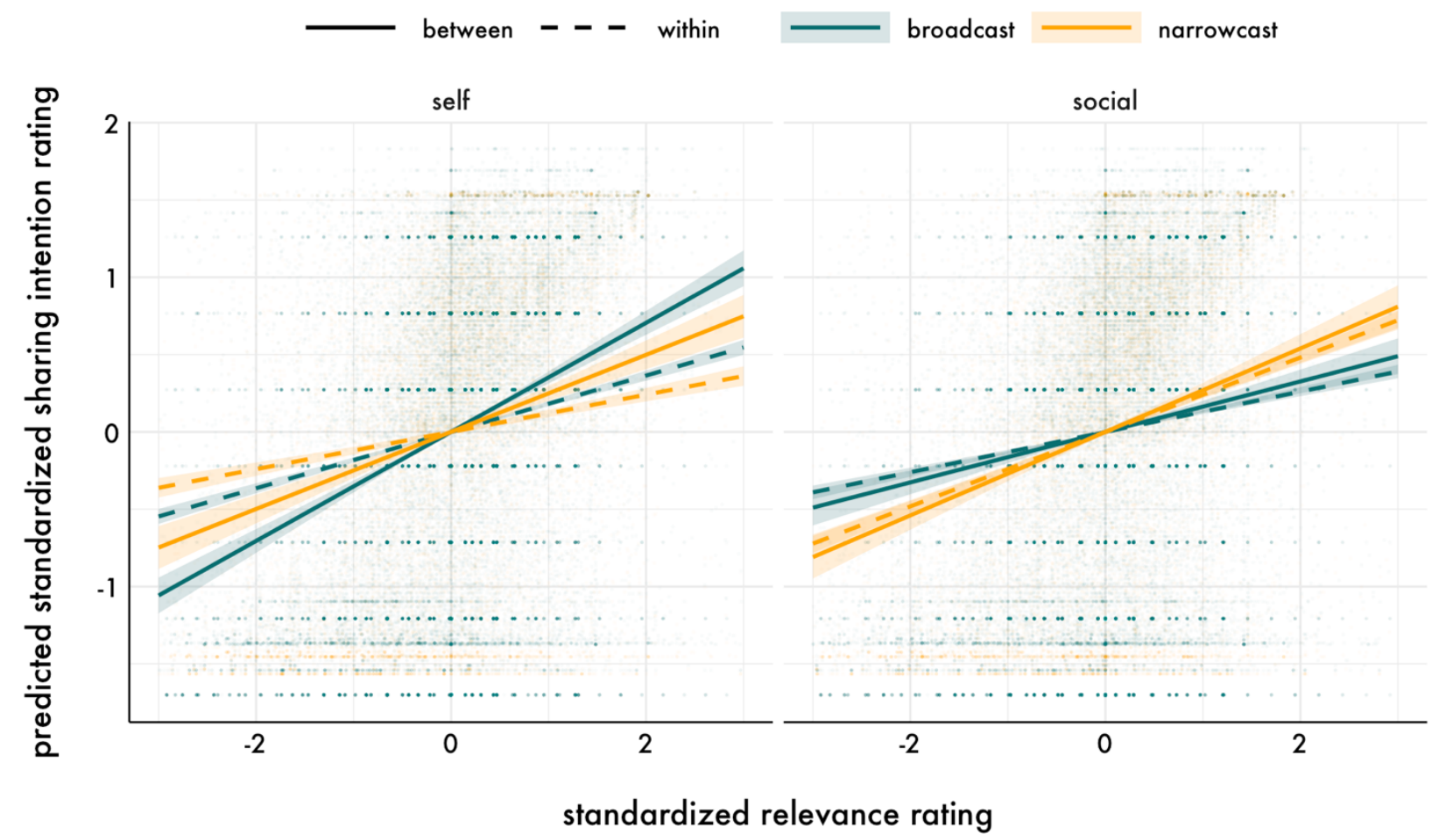

Figure 1. The predicted within- and between-person relationships for relevance ratings and sharing intention ratings from the mega-analysis as a function of within- and between-person relevance variable (self or social) and sharing type (broad- or narrowcasting). The points represent the raw (i.e., not predicted) message-level responses; error bands are 95\% confidence intervals. This plot shows that all variables are positively related to sharing intentions. The left panel visualizes the relationships between sharing intentions and self-relevance, and shows that the relationship with sharing intentions is stronger when broadcasting compared to narrowcasting for both within- and between-person self-relevance. The right panel visualizes the relationships between sharing intentions and social relevance, and shows that the relationship with sharing intentions is stronger when narrowcasting compared to broadcasting for within- and between-person social relevance. 
Table 3

Results from the mega-analysis model of predictors of sharing intentions

\begin{tabular}{lccccc}
\hline Parameter & $\beta[95 \% \mathrm{CI}]$ & $d$ & $d f$ & $t$ & $p$ \\
\hline Sharing type & $-0.00[-0.01,0.01]$ & 0.00 & 23772.62 & 0.01 & .990 \\
Self between & $0.35[0.31,0.39]$ & 0.58 & 3776.06 & 17.94 & $<.001$ \\
Self within & $0.18[0.17,0.20]$ & 2.46 & 325.24 & 22.16 & $<.001$ \\
Social between & $0.16[0.12,0.20]$ & 0.27 & 3743.23 & 8.33 & $<.001$ \\
Social within & $0.13[0.12,0.14]$ & 2.06 & 287.62 & 17.49 & $<.001$ \\
Self between x Sharing type & $-0.10[-0.13,-0.07]$ & 0.09 & 23690.57 & 7.08 & $<.001$ \\
Self within x Sharing type & $-0.06[-0.08,-0.04]$ & 0.11 & 13738.55 & 6.57 & $<.001$ \\
Social between x Sharing type & $0.11[0.08,0.14]$ & 0.09 & 23694.57 & 7.30 & $<.001$ \\
Social within x Sharing type & $0.11[0.09,0.13]$ & 0.22 & 11895.65 & 11.57 & $<.001$ \\
\hline
\end{tabular}

Note. "Within" parameters refer to the person-centered level-1 predictors, whereas "between" parameters refer to grand-mean centered level-2 predictors. The reference group for sharing type is broadcast sharing intentions. Coefficients are in standardized units. Degrees of freedom $(d f)$ were calculated using the Satterthwaite approximation.

\section{Specification curve analysis}

Overall, the relationships between self and social relevance, and sharing intentions, generalized across message content type, content medium, and sharing type, and were robust to the inclusion of demographic covariates. Between-person self-relevance was consistently the strongest predictor of sharing intentions after adjusting for the other relevance variables in the model (Figure 2; Table 4). Across all model specifications, between-person self-relevance $($ Median $\beta=0.46$, range $=0.22-0.74)$, and within-person self $($ Median $\beta=0.16$, range $=0.08$ 0.22 ) and social relevance (Median $\beta=0.14$, range $=0.10-0.30$ ) were positively related to sharing intentions and these relationships were statistically significant in every model. This means that people who tended to rate the messages as more relevant to themselves were also more likely to intend to share the messages, and when people rated messages as more relevant to themselves and to others they also reported higher intentions to share them. The relationship between sharing intentions and between-person social relevance was less consistent. Most models were positively related to sharing intentions (Median $\beta=0.13$, range $=-0.08-0.24$ ), but 
SHARING, SELF AND SOCIAL RELEVANCE

these relationships were only statistically significant in $63.95 \%$ of the models. Inspection of the model subsets (Table S3, Figures S3-4) showed that this was due to negative coefficients from models of broadcasting newspaper articles about COVID-19 (Study 5) and non-significant coefficients from models of broadcasting newspaper articles about climate change (Studies 5 and 6). Because studies 5 and 6 also had the highest average correlations between message-level self and social relevance (Table $\mathrm{S} 1$, mean $r \mathrm{~s}=.73-.84$ ), it seems likely that the coefficients from these models were less stable due to multicollinearity (see Supplementary Material for separate models).

The specification curves also revealed two interesting dissociations between self and social relevance. First, the relationship between sharing intentions and between-person selfrelevance was consistently stronger for newspaper articles compared to social media messages (collapsed across content type), whereas between-person social relevance tended to be more strongly associated with sharing intentions for social media messages (Figure 3A-B; Table S3). Second, within-person self-relevance tended to be more strongly associated with broadcast sharing intentions than narrowcast sharing intentions, whereas the opposite was true for withinperson social relevance (Figure 3C-D; Table S3). 

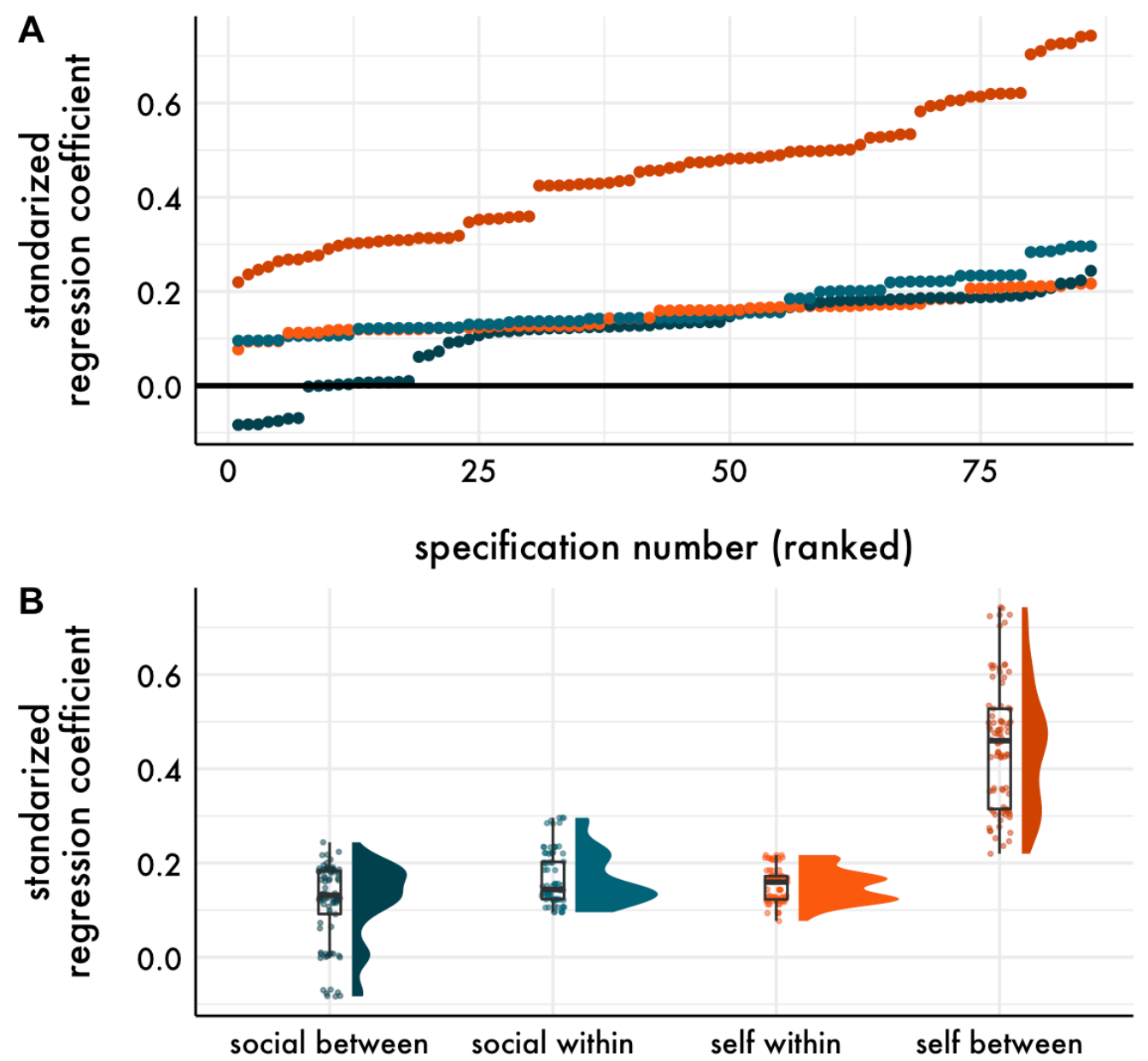

Figure 2. Specification curve comparison. (A) The top panel shows separate specification curves for each relevance variable. Within each curve, models are ordered by the magnitude of the standardized regression coefficient. (B) The bottom panel shows the distribution of standardized regression coefficients in the curve and box and whisker plots depicting the curve median (the horizontal line), the interquartile range (the box), and +/- 1.5 times the interquartile range from the box hinge (the vertical lines), for each relevance variable separately.

Table 4

Specification curve descriptives statistics

\begin{tabular}{lcccc}
\hline Parameter & Median $\beta$ & $\beta$ Range & Positive \& significant & Negative \& significant \\
\hline Self between & 0.46 & $0.22,0.74$ & $100 \%$ & $0 \%$ \\
Self within & 0.16 & $0.08,0.22$ & $100 \%$ & $0 \%$ \\
Social between & 0.13 & $-0.08,0.24$ & $64 \%$ & $0 \%$ \\
Social within & 0.14 & $0.10,0.30$ & $100 \%$ & $0 \%$ \\
\hline
\end{tabular}

Note. This information is further broken down by sharing type and message medium in Table S3. 


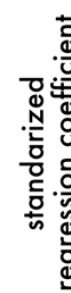

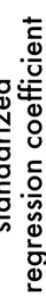
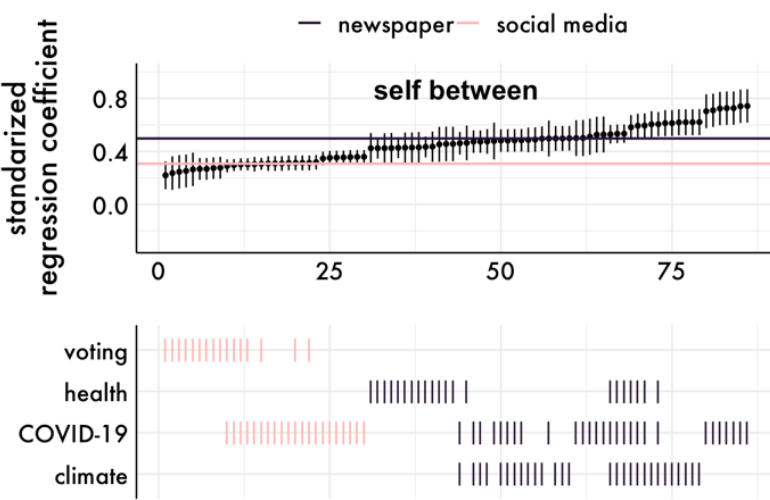

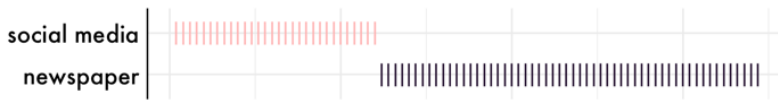

narrowcast | | || ||| | | ||||||||||||||||||||||

broadcast | H|HHHHHHHHHH|HH|H| | | ||| | ||||||||||||||||||||||

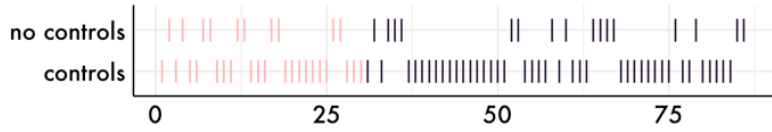

C
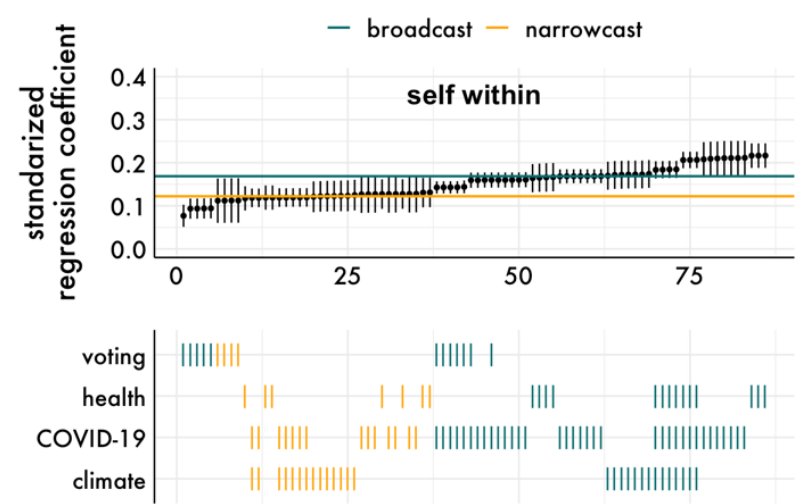

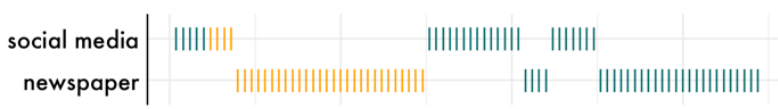

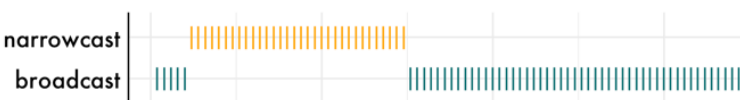

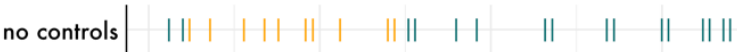

\begin{tabular}{c} 
controls \\
\cline { 2 - 3 }
\end{tabular}

specifications (ranked)
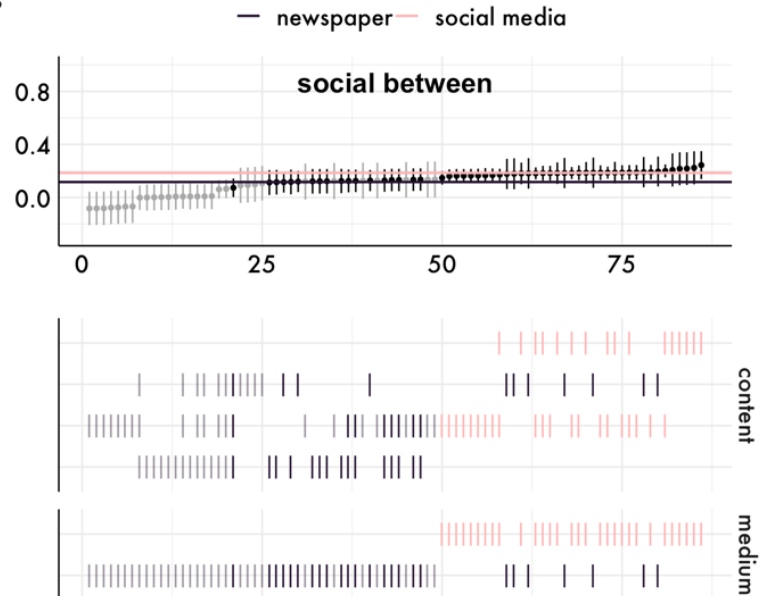

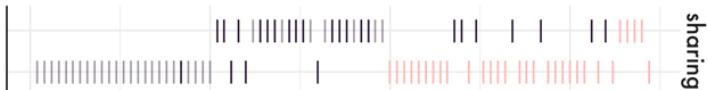

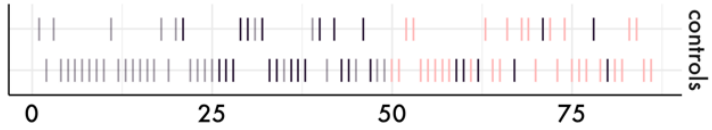

specifications (ranked)

D

- broadcast - narrowcast
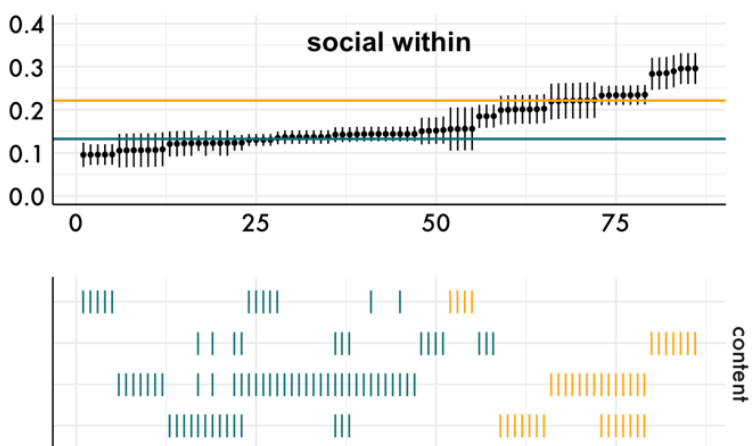

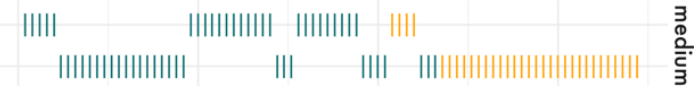

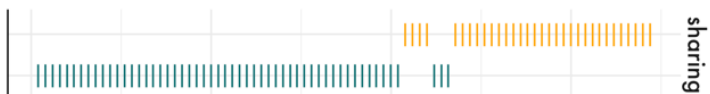

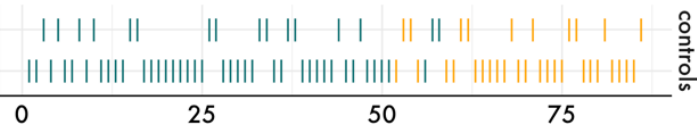

specifications (ranked)

Figure 3. Specification curve visualizing the relationships between sharing intentions and (A) betweenperson self-relevance, (B) between-person social relevance, (C) within-person self-relevance, and (D) within-person social relevance, across analytic decisions and subsets of the data. The top panels depict the relationship between the relevance variables and sharing intentions. Each dot represents the standardized regression coefficient for the relevance variable of interest from a unique model specification with a 95\% confidence interval around it. Model specifications are ordered by the regression coefficient; models for which the regression coefficient of interest was statistically significant at $p<.05$ are visualized in black, whereas coefficients $p>.05$ are in gray. The colored horizontal lines represent the median regression 
coefficient across model specifications for each relevance variable, separately. The bottom panels show the analytic decisions that were included in each model specification. Model specifications for betweenperson variables (A \& B) are colored based on message medium, whereas they are colored based on sharing type for within-person variables $(C \& D)$. Models for which the regression coefficient of interest was statistically significant at $p<0.05$ are visualized are opaque, whereas coefficients $p>0.05$ are partially opaque. Content $=$ content type medium $=$ message medium; sharing $=$ sharing type; controls $=$ inclusion of demographic covariates.

\section{Causal experiment analyses}

We extend the correlational findings by testing whether self and social relevance are causally related to sharing intentions in a preregistered experiment. Self and social relevance were experimentally manipulated by having participants explicitly reflect on the self or social relevance of messages.

\section{Methods}

\section{Participants}

This preregistered study was conducted online through MTurk. Participants were included if they were adults 18 or older, residing in the United States, were fluent in English, and passed an initial attention screening question. Participants were excluded based on the standard operating procedures for this project. Of the 644 participants initially recruited, participants were excluded for failing the English comprehension question $(n=20)$, one or more attention check $(n$ $=80)$, or for not providing comprehensible text during the experimental manipulation $(n=233)$, which was evaluated by two researchers before any hypothesis testing, consistent with our preregistered plan. This yielded a final sample of 397.

\section{Procedure}

Participants were randomly assigned to either the self $(n=200)$ or social $(n=197)$ condition. We used a mixed design in which all participants saw a set of 5 messages in the control condition and a set of 5 messages either in the self condition or the social condition. Therefore, relationships between the experimental condition (self or social) and the control 
SHARING, SELF AND SOCIAL RELEVANCE

condition were assessed within-person, whereas the difference between experimental conditions was assessed between-person. We manipulated self relevance by asking participants to write about why the article matters to them personally (self condition), and social relevance by asking them to write about why the article matters to people they know (social condition). In the control condition, participants did not reflect on relevance and instead were asked to write what the article is about. Messages consisted of a news headline and brief abstract from the New York Times about general health or climate change - two important societal issues that could benefit from individual and collective action. Participants rated self and social relevance, and broad- and narrowcast sharing intentions in a similar manner as the Studies 1-5 (see Supplementary Material). For further methodological details, including the full instructions for the task, see Supplementary Material.

\section{Statistical analyses}

First, we conducted two manipulation checks to confirm that the experimental manipulations increased self and social relevance compared to the control condition. In separate multilevel models, we regressed self or social relevance ratings on the experimental condition (self, social, or control), and the control condition was specified as the reference. The intercept and condition slope were allowed to vary randomly across participants. Next, we tested the hypothesis that the experimental manipulations would increase message sharing intentions relative to the control condition using multilevel modeling, and also tested whether the relationship between condition and sharing intention was moderated by sharing audience (broador narrowcast). We regressed sharing intentions on condition, sharing type, and their interaction, and allowed the intercept and sharing audience to vary randomly across participants (which was the least constrained model that converged). Standard effect sizes were computed using the 
SHARING, SELF AND SOCIAL RELEVANCE

Ime.dscore function from the EMAtools packages (Version 0.1.4; Kleiman, 2021). Finally, we fit four within-person Bayesian mediation models testing the degree to which the effect of the experimental condition (self v. control, or social v. control) on sharing intentions was mediated by self and social relevance, separately for broadcasting and narrowcasting. Self and social relevance were included as parallel mediators (see Supplementary Material for separate models). Intercepts and experimental condition were allowed to vary randomly across people. The raw units were retained here (versus standardizing) to facilitate interpretation in meaningful units. The mediation models were estimated using the brm function from the brms package (Bürkner, 2017) in R with the default, flat prior. Intervals around the path estimates are $95 \%$ credibility intervals from the posterior distribution.

\section{Results}

\section{Manipulation checks}

Here we tested whether the self and social experimental conditions increased self and social relevance, respectively, compared to the control condition. As expected, the self condition elicited higher self-relevance ratings compared to the control condition $(b=12.41,95 \% \mathrm{CI}$ $[10.02,14.79)$, and the social condition elicited higher social relevance ratings than the control condition $(b=8.90,95 \% \mathrm{CI}[6.82,10.99])$. We also found that the self condition increased social relevance ratings and the social condition increased self-relevance ratings (Figure 4A; Table 5).

Table 5

Results from the manipulation check models

\begin{tabular}{lcccccc}
\hline Model & Condition & $b[95 \% \mathrm{CI}]$ & $d$ & $d f$ & $t$ & $p$ \\
\hline Self-relevance & Control (intercept) & $52.85[50.55,55.14\rceil$ & - & 396.00 & 45.13 & $<.001$ \\
& Self v. Control & $12.41[10.02,14.79]$ & 1.36 & 225.44 & 10.19 & $<.001$ \\
& Social v. Control & $5.12\lceil 2.97,7.27\rceil$ & 0.64 & 212.74 & 4.67 & $<.001$ \\
Social relevance & Control (intercept) & $58.44[56.19,60.69]$ & - & 396.00 & 50.88 & $<.001$ \\
& Self v. Control & $8.66[6.62,10.69]$ & 1.10 & 228.12 & 8.32 & $<.001$ \\
& Social v. Control & $8.90[6.82,10.99]$ & 1.13 & 220.99 & 8.38 & $<.001$ \\
\hline
\end{tabular}

Note. Coefficients are in raw, unstandardized units. Degrees of freedom $(d f)$ were calculated using the 
A

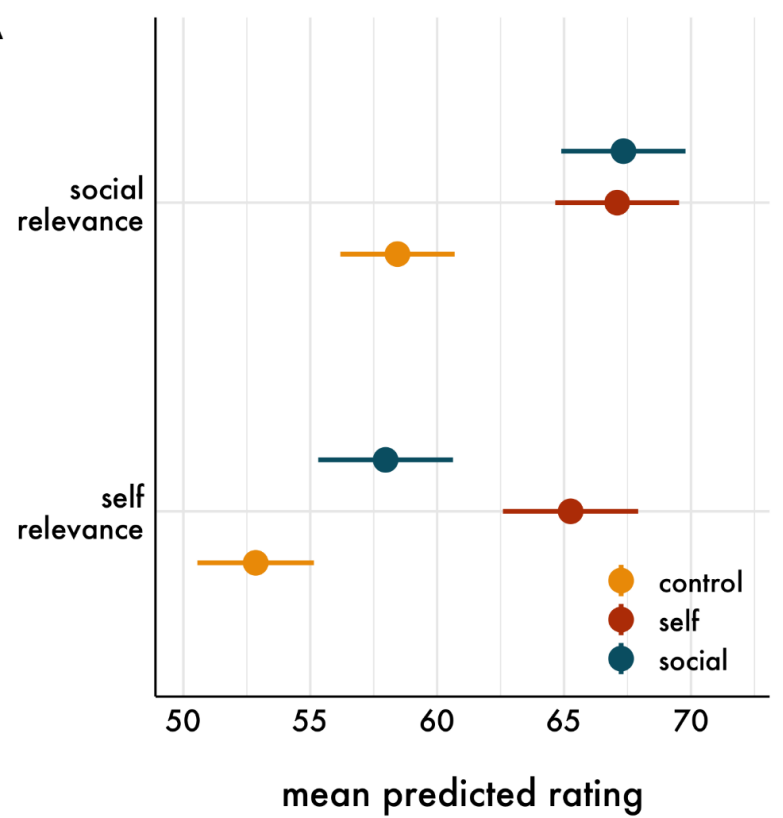

B

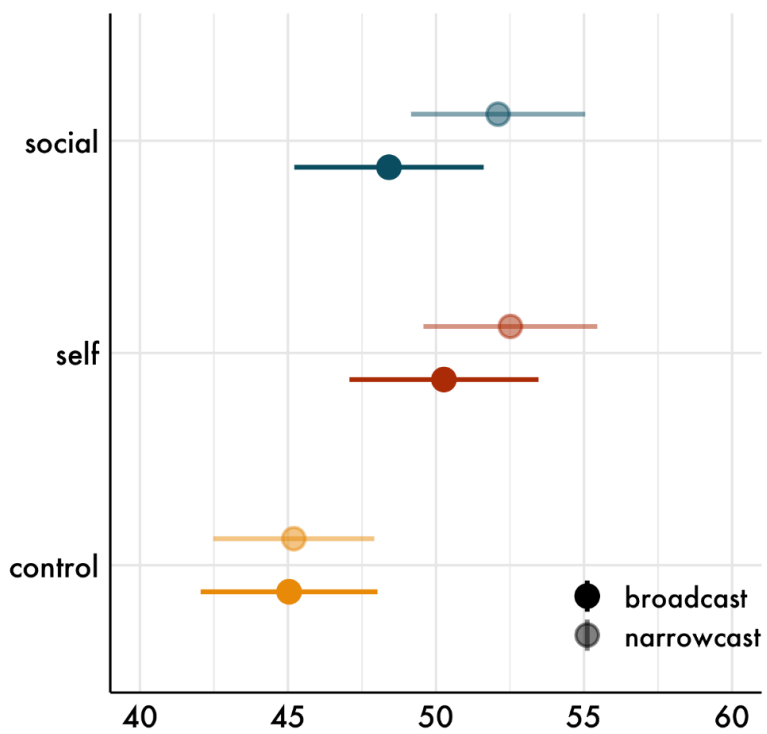

mean predicted sharing intention rating

Figure 4. (A) Manipulation check: Mean predicted self and social relevance ratings as a function of experimental condition (self, social, or control). (B) Effects of self- and social-relevance on sharing: Mean predicted sharing intention ratings as a function of experimental condition and sharing type (broador narrowcasting). Error bars are $95 \%$ confidence intervals.

\section{Condition effects by sharing type}

Next, we tested whether the experimental conditions increased sharing intentions. As expected, both the self $(b=5.23,95 \%$ CI $[3.57,6.89])$ and social $(b=3.37,95 \%$ CI $[1.70,5.05])$ experimental conditions were associated with stronger broadcast sharing intentions than the control condition (Figure 4B; Table 6). Directly comparing whether the effects differed as a function of sharing type revealed that the social condition had a stronger effect on narrowcasting compared to broadcasting $(b=3.53,95 \%$ CI $[1.25,5.80])$ as predicted. Although we hypothesized that the self condition would have a stronger effect on broadcasting compared to narrowcasting, this was not the case. Instead, there was a non-significant effect in the opposite direction $(b=2.08,95 \%$ CI $[-0.18,4.35])$. 
SHARING, SELF AND SOCIAL RELEVANCE

Table 6

Results from the experimental condition by sharing type model

\begin{tabular}{lccccc}
\hline Parameter & $b[95 \% \mathrm{CI}]$ & $d$ & $d f$ & $t$ & $p$ \\
\hline Control condition (intercept) & $45.04[42.05,48.03]$ & - & 431.34 & 29.54 & $<.001$ \\
Self v. Control condition & $5.23[3.57,6.89]$ & 0.14 & 7536.07 & 6.16 & $<.001$ \\
Social v. Control condition & $3.37[1.70,5.05]$ & 0.09 & 7535.21 & 3.95 & $<.001$ \\
Sharing type & $0.16[-1.49,1.81]$ & 0.01 & 743.49 & 0.19 & .850 \\
Self condition x Sharing type & $2.08[-0.18,4.35]$ & 0.04 & 6961.88 & 1.81 & .070 \\
Social condition x Sharing type & $3.53[1.25,5.80]$ & 0.07 & 6926.66 & 3.04 & $<.001$ \\
\hline
\end{tabular}

Note. Coefficients are in raw, unstandardized units. Degrees of freedom $(d f)$ were calculated using the Satterthwaite approximation. The reference group is control for condition and broadcasting for sharing type.

\section{Mediation}

Finally, we tested whether the effects of the experimental conditions on sharing intentions were mediated by within-person changes in self and social relevance. For the self condition (Figure 5A), 60\% of the total effect on broadcast sharing intentions was mediated by changes in self-relevance and $32 \%$ was mediated by changes in social relevance; $38 \%$ of the total effect on narrowcast sharing intentions was mediated by changes in self-relevance and $51 \%$ was mediated by changes in social relevance. For the social condition (Figure $5 \mathrm{~B}$ ), $35 \%$ of the total effect on broadcast sharing intentions was mediated by changes in self-relevance and $67 \%$ was mediated by changes in social relevance; $9 \%$ of the total effect on narrowcast sharing intentions was mediated by changes in self-relevance and 54\% was mediated by changes in social relevance. 


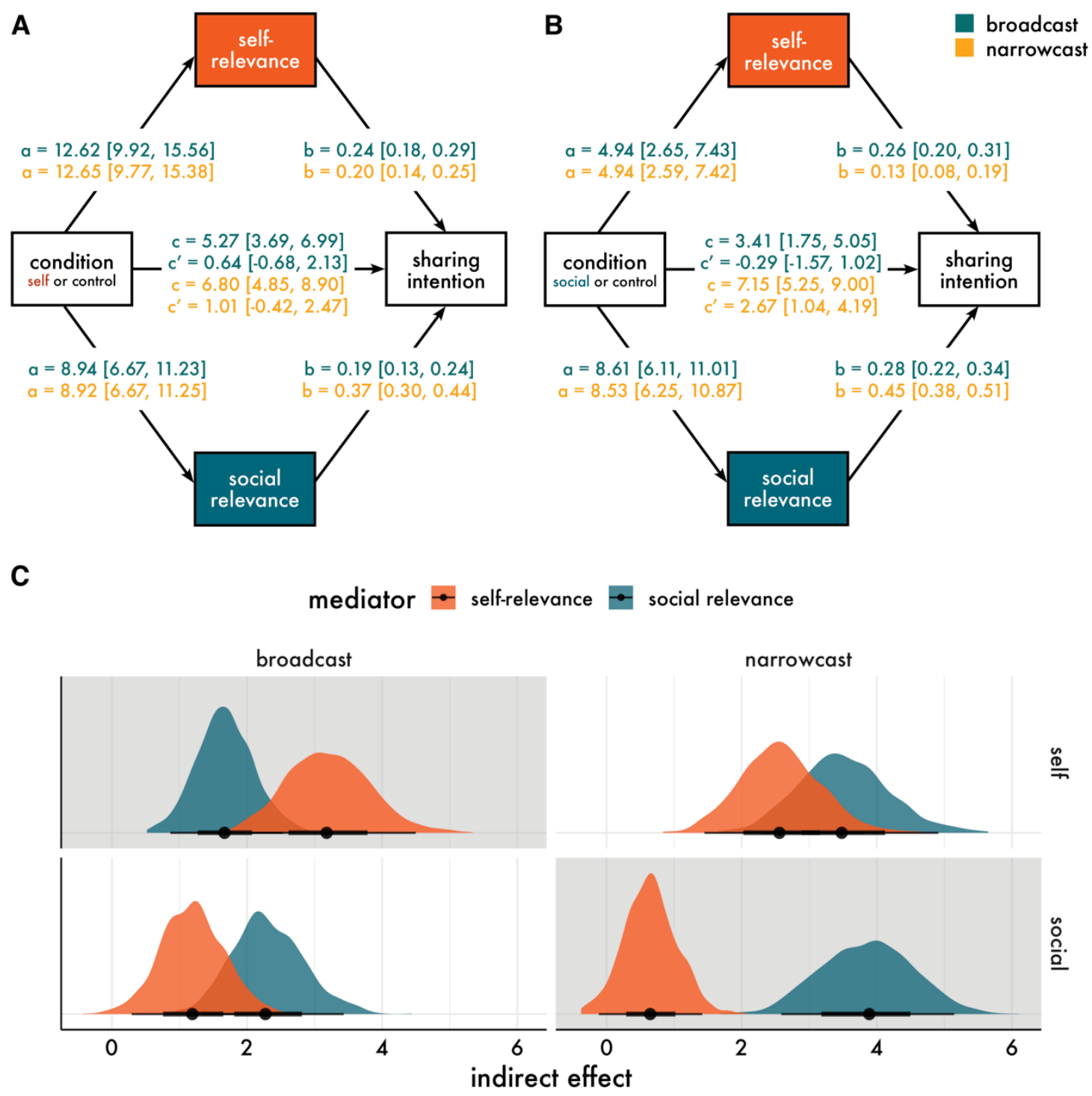

Figure 5. Results from the Bayesian mediation analyses. (A-B) Path diagrams of the within-person multilevel mediation models for the (A) self condition and (B) social condition. Parameter estimates and bootstrapped $95 \%$ credible intervals are reported for broadcast and narrowcast sharing intentions separately. $\mathrm{c}=$ total effect (direct + indirect effect of condition on sharing intention); $\mathrm{c}^{\prime}=$ direct effect. $(\mathrm{C})$ Posterior distributions for the indirect effects of the self (top panel) and social (bottom panel) conditions on broadcast (left panel) and narrowcast (right panel) sharing intentions. The gray panels highlight where the relationships between self and social relevance and sharing intentions are expected theoretically to be most differentiable. Indirect effects were calculated as $a * b+\operatorname{cov}(a, b)$. The point intervals represent the median of the posterior distribution, and the $66 \%$ and $95 \%$ credible intervals around the median.

\section{Discussion}

Information transmission within social networks supports widespread attitudinal and behavioral change. The perceived self and social relevance of the information are two 
psychological factors that may increase the value of sharing information with others. Across six studies including a wide variety of messages about pressing and potentially polarizing societal issues - COVID-19, voting, general health, and climate change-we found robust positive correlational relationships between message self and social relevance, and sharing intentions, both within- and between-person. Correlationally, self-relevance was more strongly related to intentions to share on with a wide audience (broadcasting) than directly with individual people (narrowcasting), whereas social relevance was more strongly related to narrowcasting intentions. The specification curve analysis indicated that these relationships generalized across message content and medium, and were not systematically affected by the inclusion of demographic variables. Finally, the preregistered experimental study provided evidence that self and social relevance are causally related to sharing intentions. Within-person mediation analyses showed clear specificity for the strength of the mediation effects. Consistent with prior theorizing (Barasch \& Berger, 2014), the causal effect of the self manipulation on broadcasting was more strongly mediated by self than to social relevance, whereas the effect of the social manipulation on narrowcasting was more strongly mediated by social than self-relevance. However, our data also support the idea that self- and social-relevance are related to one another, such that manipulating one increases the other. Together, these findings extend existing models of information sharing, highlight self and social relevance as important sources of value that motivate sharing, and suggest that self and social relevance can be targeted by interventions to promote information sharing across various contexts.

\section{Self and social relevance are each robustly related to sharing intentions}

Disaggregating within- and between-person relationships indicated that 1) people who think messages are more self and socially relevant also tend to report higher sharing intentions, 
and 2) when people perceive messages as more self and socially relevant, they tend to report higher intentions to share them. The direction of these relationships was consistent across different message content domains, mediums, and sharing audiences. With the exception of a set of models estimating the relationship between broadcast sharing intentions and between-person social relevance including newspaper articles about COVID-19 in Study 5, the regression coefficients in all model specifications in the specification curve analysis were positive, indicating strong consistency. Although previous studies did not distinguish within- and between-person relationships, these findings are consistent with the value-based virality model which posits self and social relevance as key factors in decisions to share (Scholz et al., 2017). They also demonstrate that despite being intimately intertwined (Ellemers et al., 2002; Harter, 1999; Scholz, Baek, et al., 2020), self and social relevance each contribute uniquely as well to sharing intentions.

\section{Experimentally manipulating self and social relevance increases sharing intentions}

Extending these correlational findings, we observed evidence that self and social relevance are causally related to sharing intentions. Reflecting on both the self and social relevance of messages increased sharing intentions, compared to a control condition. This affirms the potential of self and social relevance frames as viable intervention targets to promote sharing behavior. We examined the underlying mechanism of these interventions using withinperson mediation analyses, including perceived self and social relevance as parallel mediators. Consistent with prior work highlighting the dual roles of self and social relevance in sharing behavior (Scholz, Baek, et al., 2020), sharing intentions were partially mediated through both self and social relevance in all models. These results demonstrate that reflecting on the self and 
social relevance of content can increase sharing behavior through multiple pathways without altering the content of the messages.

\section{Relative contributions of self and social relevance depend on the sharing context}

Previous research has suggested that various motives affect decisions to share (Cappella et al., 2015; Lee \& Ma, 2012) and their relative importance depends on the sharing context (Barasch \& Berger, 2014). In the correlational analyses, self-relevance was more strongly related to broadcast compared to narrowcast sharing intentions, whereas the opposite was true for social relevance. This is in line with theoretical models emphasizing self-expression and enhancement as motives when sharing broadly and other-focused motives, such as helping and connecting, when sharing narrowly (Barasch \& Berger, 2014). However, both self and social relevance were positively and uniquely related to broad- and narrowcast sharing intentions suggesting that they are both implicated in sharing regardless of context. The within-person mediation analyses further support this idea. There were indirect effects of the experimental manipulations on sharing intentions through both self and social relevance, but that their relative strength differed by the audience size. Specifically, the proportion of the causal effect of the self manipulation on broadcasting was mediated more strongly $(\sim 2 \mathrm{x})$ for self compared to social relevance, and the proportion of the causal effect of the social manipulation on narrowcasting was mediated more strongly $(\sim 6 \mathrm{x})$ for social compared to self relevance. Together, these findings are consistent with models that treat self-related and social motives as parallel processes that both contribute to sharing decisions, but to differing degrees depending on the sharing target (Scholz, Baek, et al., 2020). 
SHARING, SELF AND SOCIAL RELEVANCE

\section{Limitations and future directions}

Despite notable strengths, such as the inclusion of large samples of people and messages, assessment of generalizability on several dimensions, and the use of correlational and causal methods, there are several limitations. First, all data were collected online. Concerns about data quality are mitigated by the relatively strict quality assurance procedures (detailed in Supplementary Material) used in these studies. Second, we did not recruit nationally or internationally representative samples. Our sample included participants from at least 49 states and was similar to the composition of adults in the United States with respect to age. However, our sample included more men, had a higher proportion of people who identified as White and Asian, and a lower proportion who identified as Black or African American, and as Hispanic or Latinx than the U.S. as a whole. Our sample also reported higher educational attainment and lower household incomes than the U.S. as a whole. Although the specification curve analysis showed that inclusion of these demographic variables did not systematically alter the strength of the relationships, future work should be designed to explicitly examine demographic, crossnational, political, and cross-cultural influences. Third, these studies focused on self-reported sharing intentions. Intentions are important precursors of behavior (Albarracin et al., 2021), but it would be useful to test these relationships in the context of actual sharing behavior. Fourth, although we experimentally manipulated self and social relevance and examined mediation within-person, it is possible that unmeasured variables influenced the observed results. Finally, these analyses do not take into account individual differences, such as political orientation or social network configuration, or message properties, such as emotionality or concreteness, that might moderate the relationships between self and social relevance and sharing intentions. These 
SHARING, SELF AND SOCIAL RELEVANCE

are important future directions and we have shared the messages used in these studies to enable other researchers to explore message-level characteristics in these data.

\section{Conclusions and translational implications}

Across six studies, including nearly thirty-one thousand message ratings about critical societal issues, we demonstrated correlational and causal evidence that perceived message self and social relevance are positively related to intentions to share content online. We conducted these analyses in ways that promote replicability and generalizability in order to maximize the translational potential of these findings, including: preregistering our hypotheses and analysis plans in Studies 5 and 6, aggregating across studies using the least constrained random effects structure possible, exploring the stability of the relationships using specification curve analysis, and using experimental manipulation to test causal relationships. Overall, this work suggests multiple viable routes to increasing information transmission, including: recruiting individuals who perceive the content as self or socially relevant to serve as messengers, tailoring messages to be more self or socially relevant to individuals, and intervening to draw attention to message self or social relevance without changing the message content itself, similar to recent interventions that shift attention to information accuracy to decrease sharing misinformation (And1 \& Akesson, 2020; Pennycook et al., 2021). Such message framing approaches are particularly promising as scalable interventions since they only require a message and a prompt to reflect on relevance, and such prompts could readily be offered (e.g., by activists, government agencies, news organizations) to promote information sharing and catalyze action (Barberá et al., 2015). Together, this work extends existing theories of information sharing and provides compelling evidence that self and social relevance are important psychological factors that influence 
SHARING, SELF AND SOCIAL RELEVANCE

decisions to share information that can be leveraged to promote attitudinal and behavioral change.

\section{Acknowledgements}

We thank the following people for helping develop the stimuli used in Studies 1-3: Rui Pei; Annie Cheng, Benjamin Ai, Benjamin Oh, Ellen Manford, Harrison Feinman, Omotoyosi Abu, Shivani Guha, Siddharth Jaiswal, Cory Bowman, and other collaborators from Penn Leads the Vote; Eytan Oren, Caitlin Gray, and other collaborators from the 100 Million Project. EBF has consulted within the past year for Google and serves on the scientific advisory board for Kumanu, a digital well-being company. DC consulted within the past year for Lotic, a digital behavior change company.

\section{Positionality statement}

In acknowledgement that our identities can influence our approach to science (Roberts et al., 2020) the authors wish to provide the reader with information about our backgrounds. With respect to gender, when the manuscript was drafted, 6 authors self-identified as women and 4 authors as men. With respect to race and ethnicity, 1 author self-identified as Chinese, 1 author as South Asian, 7 authors as White, and 1 author as White Hispanic. With respect to age, all authors are 40 years old or younger.

\section{Citation diversity statement}

Recent work in several fields of science has identified a bias in citation practices such that papers from women and other minority scholars are under-cited relative to the number of such papers in the field (Bertolero et al., 2020; Caplar et al., 2017; Chatterjee \& Werner, 2021; Dion et al., 2018; Dworkin et al., 2020; Fulvio et al., 2021; Maliniak et al., 2013; Mitchell et al., 2013; Wang et al., n.d.). Here we sought to proactively consider choosing references that reflect 
the diversity of the field in thought, form of contribution, gender, race, ethnicity, and other factors. First, we obtained the predicted gender of the first and last author of each reference (excluding software package citations) by using databases that store the probability of a first name being carried by a woman (Caplar et al., 2017; Dion et al., 2018; Dworkin et al., 2020; Maliniak et al., 2013; Mitchell et al., 2013; Zhou et al., 2020). By this measure (and excluding self-citations to the first and last authors of our current paper), our references contain $21 \%$ woman(first)/woman(last), 13\% man/woman, 30\% woman/man, and 36\% man/man. This method is limited in that a) names, pronouns, and social media profiles used to construct the databases may not, in every case, be indicative of gender identity and b) it cannot account for intersex, non-binary, or transgender people. 


\section{References}

Al-Rawi, A. (2019). Viral news on social media. Digital journalism, 7(1), 63-79.

Albarracin, D., Sunderrajan, A., McCulloch, K. C., \& Jones, C. (2021). Mistaking an Intention for a Behavior: The Case of Enacting Behavioral Decisions Versus Simply Intending to Enact Them. Personality and Social Psychology Bulletin, 47(3), 455-467. https://doi.org/10.1177/0146167220929203

And1, S., \& Akesson, J. (2020). Nudging Away False News: Evidence from a Social Norms Experiment. Digital Journalism, 9(1), 1-21. https://doi.org/10.1080/21670811.2020.1847674

Baek, E. C., Scholz, C., O’Donnell, M., \& Falk, E. B. (2017). The Value of Sharing Information: A Neural Account of Information Transmission. Psychological Science, 28(7). https://doi.org/10.1177/0956797617695073

Baek, E. C., Tamir, D., \& Falk, E. B. (2019). Considering Others' Mental States Causally Increases Feelings of Social Bonding and Information Sharing. https://doi.org/10.31234/osf.io/nw43x

Bakdash, J. Z., \& Marusich, L. R. (2017). Repeated Measures Correlation. Frontiers in Psychology, 8, 456. https://doi.org/10.3389/fpsyg.2017.00456

Barasch, A., \& Berger, J. (2014). Broadcasting and Narrowcasting: How Audience Size Affects What People Share. Journal of Marketing Research, 51(3), 286-299. https://doi.org/10.1509/jmr.13.0238

Barberá, P., Wang, N., Bonneau, R., Jost, J. T., Nagler, J., Tucker, J., \& González-Bailón, S. (2015). The Critical Periphery in the Growth of Social Protests. PLOS ONE, 10(11), e0143611. https://doi.org/10.1371/journal.pone.0143611 
SHARING, SELF AND SOCIAL RELEVANCE

Bates, D., Maechler, M., Bolker, B., \& Walker, S. (2015). Fitting Linear Mixed-Effects Models Using lme4. Journal of Statistical Software, 67(1), 1-48. https://doi.org/10.18637/jss.v067.i01.

Bates, D. \& Maechler, M. (2019). Matrix: Sparse and Dense Matrix Classes and Methods. R package version 1.2-18. https://CRAN.R-project.org/package=Matrix

Baumeister, R. F., \& Leary, M. R. (1995). The Need to Belong: Desire for Interpersonal Attachments as a Fundamental Human Motivation. Psychological Bulletin, 117(3), 497529. https://doi.org/10.1037/0033-2909.117.3.497

Beer, J. S., \& Hughes, B. L. (2010). Neural systems of social comparison and the "aboveaverage" effect. NeuroImage, 49(3), 2671-2679. https://doi.org/10.1016/j.neuroimage.2009.10.075

Berger, J. (2014). Word of mouth and interpersonal communication: A review and directions for future research. Journal of Consumer Psychology, 24(4), 586-607. https://doi.org/10.1016/j.jcps.2014.05.002

Berger, J., \& Milkman, K. L. (2012). What Makes Online Content Viral? Journal of Marketing Research, 49(2), 192-205. https://doi.org/10.1509/jmr.10.0353

Berkman, E. T., Livingston, J. L., \& Kahn, L. E. (2017). Finding The "Self” in Self-Regulation: The Identity-Value Model. Psychological Inquiry, 28(2-3), 77-98. https://doi.org/10.1080/1047840x.2017.1323463

Bertolero, M. A., Dworkin, J. D., David, S. U., Lloreda, C. L., Srivastava, P., Stiso, J., Zhou, D., Dzirasa, K., Fair, D. A., Kaczkurkin, A. N., Marlin, B. J., Shohamy, D., Uddin, L. Q., Zurn, P., \& Bassett, D. S. (2020). Racial and ethnic imbalance in neuroscience reference 
SHARING, SELF AND SOCIAL RELEVANCE

lists and intersections with gender. BioRxiv, 2020.10.12.336230.

https://doi.org/10.1101/2020.10.12.336230

Bolker, B. \& Robinson, D. (2021). broom.mixed: Tidying Methods for Mixed Models. R package version 0.2.7. https://CRAN.R-project.org/package=broom.mixed

Bürkner, P. C. (2017). brms: An R package for Bayesian multilevel models using Stan. Journal of Statistical Software, 80, 1-28.

Canty, A., \& Ripley, B. (2019). boot: Bootstrap R (S-Plus) Functions. R package version 1.3-24.

Caplar, N., Tacchella, S., \& Birrer, S. (2017). Quantitative evaluation of gender bias in astronomical publications from citation counts. Nature Astronomy, 1(6), 0141. https://doi.org/10.1038/s41550-017-0141

Cappella, J. N., Kim, H. S., \& Albarracín, D. (2015). Selection and Transmission Processes for Information in the Emerging Media Environment: Psychological Motives and Message Characteristics. Media Psychology, 18(3), 396-424.

https://doi.org/10.1080/15213269.2014.941112

Chatterjee, P., \& Werner, R. M. (2021). Gender Disparity in Citations in High-Impact Journal Articles. JAMA Network Open, 4(7), e2114509. https://doi.org/10.1001/jamanetworkopen.2021.14509

Chavez, R. S., Heatherton, T. F., \& Wagner, D. D. (2017). Neural population decoding reveals the intrinsic positivity of the self. Cerebral Cortex, 27(11), 5222-5229.

Cialdini, R. B., Kallgren, C. A., \& Reno, R. R. (1991). A focus theory of normative conduct: A theoretical refinement and reevaluation of the role of norms in human behavior. In Advances in experimental social psychology (Vol. 24, pp. 201-234). Academic Press. 
SHARING, SELF AND SOCIAL RELEVANCE

Cosme, D., Scholz, C., Chan, H. Y., Dore, B. P., Tartak, J. C., Burns, S., \& Falk, E. B. (2022, April 13). Message self and social relevance increases intentions to share content: Correlational and causal evidence from six studies. https://doi.org/10.17605/OSF.IO/BGS5Y

D’Argembeau, A. (2013). On the Role of the Ventromedial Prefrontal Cortex in Self-Processing: The Valuation Hypothesis. 7. https://doi.org/10.3389/fnhum.2013.00372

Dion, M. L., Sumner, J. L., \& Mitchell, S. M. (2018). Gendered Citation Patterns across Political Science and Social Science Methodology Fields. Political Analysis, 26(3), 312-327. https://doi.org/10.1017/pan.2018.12

Doré, B. P., Scholz, C., Baek, E. C., Garcia, J. O., O’Donnell, M. B., Bassett, D. S., Vettel, J. M., \& Falk, E. B. (2019). Brain Activity Tracks Population Information Sharing by Capturing Consensus Judgments of Value. Cerebral Cortex, 29(7), 3102-3110. https://doi.org/10.1093/cercor/bhy176

Dworkin, J. D., Linn, K. A., Teich, E. G., Zurn, P., Shinohara, R. T., \& Bassett, D. S. (2020). The extent and drivers of gender imbalance in neuroscience reference lists. Nature Neuroscience, 23(8), 918-926. https://doi.org/10.1038/s41593-020-0658-y

Echterhoff, G., Lang, S., Krämer, N., \& Higgins, E. T. (2009). Audience-Tuning Effects on Memory: The Role of Audience Status in Sharing Reality. Social Psychology, 40(3), 150-163. https://doi.org/10.1027/1864-9335.40.3.150

Eisenhauer, J. G. (2021). Meta-analysis and mega-analysis: A simple introduction. Teaching Statistics, 43(1), 21-27. https://doi.org/10.1111/test.12242

Ellemers, N., Spears, R., \& Doosje, B. (2002). Self and social identity. Annual Review of Psychology, 53(1), 161-186. https://doi.org/10.1146/annurev.psych.53.100901.135228 
SHARING, SELF AND SOCIAL RELEVANCE

Falk, E. B., Berkman, E. T., \& Lieberman, M. D. (2012). From Neural Responses to Population Behavior. Psychological Science, 23(5), 439-445. https://doi.org/10.1177/0956797611434964

Falk, E. B., \& Scholz, C. (2018). Persuasion, Influence, and Value: Perspectives from Communication and Social Neuroscience. Annual Review of Psychology, 69(1), 1-28. https://doi.org/10.1146/annurev-psych-122216-011821

Fulvio, J. M., Akinnola, I., \& Postle, B. R. (2021). Gender (Im)balance in Citation Practices in Cognitive Neuroscience. Journal of Cognitive Neuroscience, 33(1), 3-7. https://doi.org/10.1162/jocn_a_01643

Genevsky, A., \& Knutson, B. (2015). Neural Affective Mechanisms Predict Market-Level Microlending. Psychological Science, 26(9), 1411-1422. https://doi.org/10.1177/0956797615588467

Giudice, M. D., \& Gangestad, S. W. (2021). A Traveler's Guide to the Multiverse: Promises, Pitfalls, and a Framework for the Evaluation of Analytic Decisions. Advances in Methods and Practices in Psychological Science, 4(1), 251524592095492. https://doi.org/10.1177/2515245920954925

Harter, S. (1999). The Construction of the Self: A Developmental Perspective. Guilford Publications.

Henry, L., \& Wickham, H. (2020). purrr: Functional Programming Tools. R package version 0.3.4. https://CRAN.R-project.org/package= $=$ purrr

Humphreys, G. W., \& Sui, J. (2015). Attentional control and the self: The Self-Attention Network (SAN). Cognitive Neuroscience, 7(1-4), 5-17. https://doi.org/10.1080/17588928.2015.1044427 
Ihm, J., \& Kim, E. M. (2018). The hidden side of news diffusion: Understanding online news sharing as an interpersonal behavior. new media \& society, 20(11), 4346-4365.

Jeong, M., \& Bae, R. E. (2018). The effect of campaign-generated interpersonal communication on campaign-targeted health outcomes: A meta-analysis. Health Communication, 33(8), 988-1003.

Kahneman, D., Knetsch, J. L., \& Thaler, R. H. (1991). Anomalies: The Endowment Effect, Loss Aversion, and Status Quo Bias. Journal of Economic Perspectives, 5(1), 193-206. https://doi.org/10.1257/jep.5.1.193

Kassambara, A. (2020). ggpubr: 'ggplot2' Based Publication Ready Plots. R package version 0.4.0. https://CRAN.R-project.org/package=ggpubr

Kay, M. (2022). tidybayes: Tidy Data and Geoms for Bayesian Models. doi: $10.5281 /$ zenodo. 1308151

Kim, H. S. (2015). Attracting views and going viral: How message features and news-sharing channels affect health news diffusion. Journal of Communication, 65(3), 512-534.

Kim, H. S. (2021). How message features and social endorsements affect the longevity of news sharing. Digital Journalism, 9(8), 1162-1183.

Kleiman (2021). EMAtools: Data Management Tools for Real-Time Monitoring/Ecological Momentary Assessment Data. R package version 0.1.4. https://CRAN.Rproject.org/package $=$ EMAtools

Koster-Hale, J., \& Saxe, R. (2013). Theory of Mind: A Neural Prediction Problem. Neuron, 79(5), 836-848. https://doi.org/10.1016/j.neuron.2013.08.020 
SHARING, SELF AND SOCIAL RELEVANCE

Kuznetsova, A., Brockhoff, P. B., \& Christensen, R. H. B. (2017). lmerTest Package:Tests in Linear Mixed Effects Models. Journal of Statistical Software, 82(13), 1-26. https://doi.org/10.18637/jss.v082.i13

Kümpel, A. S., Karnowski, V., \& Keyling, T. (2015). News sharing in social media: A review of current research on news sharing users, content, and networks. Social media+ society, 1(2), 2056305115610141.

Lee, C. S., \& Ma, L. (2012). News sharing in social media: The effect of gratifications and prior experience. Computers in Human Behavior, 28(2), 331-339. https://doi.org/10.1016/j.chb.2011.10.002

Levy, D. J., \& Glimcher, P. W. (2012). The root of all value: a neural common currency for choice. Current Opinion in Neurobiology, 22(6), 1027-1038. https://doi.org/10.1016/j.conb.2012.06.001

Maliniak, D., Powers, R., \& Walter, B. F. (2013). The Gender Citation Gap in International Relations. International Organization, 67(4), 889-922. https://doi.org/10.1017/s0020818313000209

Makowski, D., Ben-Shachar, M.S., Patil, I. \& Lüdecke, D. (2020). Automated Results Reporting as a Practical Tool to Improve Reproducibility and Methodological Best Practices Adoption. CRAN. Available from https://github.com/easystats/report Markus, H. (1977). Self-schemata and processing information about the self. Journal of Personality and Social Psychology, 35(2), 63-78. https://doi.org/10.1037/00223514.35.2.63

Markus, H. (1983). Self-knowledge: An expanded view. Journal of Personality, 51(3), 543-565. https://doi.org/10.1111/j.1467-6494.1983.tb00344.x 
SHARING, SELF AND SOCIAL RELEVANCE

Masur, Philipp K. \& Scharkow, M. (2020). specr: Conducting and Visualizing Specification Curve Analyses. Available from https://CRAN.R-project.org/package=specr

Meyer, M. L., \& Lieberman, M. D. (2018). Why People Are Always Thinking about Themselves: Medial Prefrontal Cortex Activity during Rest Primes Self-referential Processing. Journal of Cognitive Neuroscience, 30(5), 714-721. https://doi.org/10.1162/jocn_a_01232

Mezulis, A. H., Abramson, L. Y., Hyde, J. S., \& Hankin, B. L. (2004). Is There a Universal Positivity Bias in Attributions? A Meta-Analytic Review of Individual, Developmental, and Cultural Differences in the Self-Serving Attributional Bias. Psychological Bulletin, 130(5), 711-747. https://doi.org/10.1037/0033-2909.130.5.711

Mildner, J. N., \& Tamir, D. I. (2021). The People Around You Are Inside Your Head: Social Context Shapes Spontaneous Thought. Journal of Experimental Psychology: General. https://doi.org/10.1037/xge0001057

Mitchell, S. M., Lange, S., \& Brus, H. (2013). Gendered Citation Patterns in International Relations Journals. International Studies Perspectives, 14(4), 485-492. https://doi.org/10.1111/insp.12026

Pennycook, G., Epstein, Z., Mosleh, M., Arechar, A. A., Eckles, D., \& Rand, D. G. (2021). Shifting attention to accuracy can reduce misinformation online. Nature, 1-6. https://doi.org/10.1038/s41586-021-03344-2

Pfeifer, J. H., \& Berkman, E. T. (2018). The Development of Self and Identity in Adolescence: Neural Evidence and Implications for a Value-Based Choice Perspective on Motivated Behavior. Child Development Perspectives, 12(3), 158-164. https://doi.org/10.1111/cdep.12279 
Pfeifer, J. H., \& Peake, S. J. (2012). Self-development: Integrating cognitive, socioemotional, and neuroimaging perspectives. 2(1). https://doi.org/10.1016/j.den.2011.07.012

Scholz, C., Baek, E. C., O’Donnell, M., \& Falk, E. B. (2020). Decision-making about broad- and narrowcasting: a neuroscientific perspective. Media Psychology, 23(1), 131-155. https://doi.org/10.1080/15213269.2019.1572522

Scholz, C., Baek, E. C., O’Donnell, M., Kim, H., Cappella, J. N., \& Falk, E. B. (2017). A neural model of valuation and information virality. Proceedings of the National Academy of Sciences, 114(11), 2881-2886. https://doi.org/10.1073/pnas.1615259114

Scholz, C., Jovanova, M., Baek, E. C., \& Falk, E. B. (2020). Media content sharing as a valuebased decision. Current Opinion in Psychology, 31, 83-88. https://doi.org/10.1016/j.copsyc.2019.08.004

Schultz, P. W., Nolan, J. M., Cialdini, R. B., Goldstein, N. J., \& Griskevicius, V. (2007). The Constructive, Destructive, and Reconstructive Power of Social Norms. Psychological Science, 18(5), 429-434. https://doi.org/10.1111/j.1467-9280.2007.01917.x

Silge, J. \& Robinson, D. (2016). tidytext: Text Mining and Analysis Using Tidy Data Principles in R. Journal of Open Source Software, 1(3). doi: 10.21105/joss.00037

Simonsohn, U., Simmons, J. P., \& Nelson, L. D. (2020). Specification curve analysis. Nature Human Behaviour, 4(11), 1208-1214. https://doi.org/10.1038/s41562-020-0912-z

Steegen, S., Tuerlinckx, F., Gelman, A., \& Vanpaemel, W. (2016). Increasing Transparency Through a Multiverse Analysis. Perspectives on Psychological Science, 11(5), 702-712. https://doi.org/10.1177/1745691616658637 
Tamir, D. I., Zaki, J., \& Mitchell, J. P. (2015). Informing Others Is Associated With Behavioral and Neural Signatures of Value. Journal of Experimental Psychology: General, 144(6), 1114-1123. https://doi.org/10.1037/xge0000122

Tamir, D. I., \& Mitchell, J. P. (2012). Disclosing information about the self is intrinsically rewarding. 109(21). https://doi.org/10.1073/pnas.1202129109

Tankard, M. E., \& Paluck, E. L. (2016). Norm perception as a vehicle for social change. Social Issues and Policy Review, 10(1), 181-211.

Taylor, S. E., \& Brown, J. D. (1988). Illusion and Well-Being: A Social Psychological Perspective on Mental Health. Psychological Bulletin, 103(2), 193-210. https://doi.org/10.1037/0033-2909.103.2.193

Thornton, M. A., Weaverdyck, M. E., \& Tamir, D. I. (2018). The social brain automatically predicts others' future mental states. Journal of Neuroscience, 39(1), 1431-18. https://doi.org/10.1523/jneurosci.1431-18.2018

Vaughan, D. and Dancho, M. (2021). furrr: Apply Mapping Functions in Parallel using Futures. R package version 0.2.2. https://CRAN.R-project.org/package=furrr

Vijayakumar, N., Flournoy, J. C., Mills, K. L., Cheng, T. W., Mobasser, A., Flannery, J. E., Allen, N. B., \& Pfeifer, J. H. (2020). Getting to know me better: An fMRI study of intimate and superficial self-disclosure to friends during adolescence. Journal of Personality and Social Psychology, 118(5), 885-899.

https://doi.org/10.1037/pspa0000182

Wang, X., Dworkin, J., Zhou, D., Stiso, J., Falk, E. B., Zurn, P., Bassett, D. S., \& Lydon-Staley, D. M. (n.d.). Gendered Citation Practices in the Field of Communication. https://doi.org/10.31234/osf.io/ywrcq 
SHARING, SELF AND SOCIAL RELEVANCE

Wickham, H. \& Hester, J. (2020). readr: Read Rectangular Text Data. R package version 1.4.0. https://CRAN.R-project.org/package=readr

Wickham, H. (2016) ggplot2: Elegant Graphics for Data Analysis. Springer-Verlag New York.

Wickham, H. (2019). stringr: Simple, Consistent Wrappers for Common String Operations. R package version 1.4.0. https://CRAN.R-project.org/package=stringr

Wickham, H. (2021). forcats: Tools for Working with Categorical Variables (Factors). R package version 0.5.1. https:/CRAN.R-project.org/package=forcats

Wickham, H. (2021). tidyr: Tidy Messy Data. R package version 1.1.3. https://CRAN.Rproject.org/package $=$ tidyr

Wickham, H., Averick, M., Bryan, J., Chang, W., McGowan, L. D. A., François, R., ... \& Yutani, H. (2019). Welcome to the Tidyverse. Journal of open source software, 4(43), 1686. doi: https://doi.org/10.21105/joss.01686

Wickham, H., François, R., Henry, L., \& Müller, K. (2021). dplyr: A Grammar of Data Manipulation. R package version 1.0.7. https:/CRAN.R-project.org/package=dplyr

Yihui Xie (2021). knitr: A General-Purpose Package for Dynamic Report Generation in R. R package version 1.31 .

Zhou, D., Cornblath, E. J., Stiso, J., Teich, E. G., Dworkin, J. D., Blevins, A. S., \& Bassett, D. S. (2020). Gender diversity statement and code notebook v1.0. https://github.com/dalejn/cleanBib

Zhu, H. (2020). kableExtra: Construct Complex Table with 'kable' and Pipe Syntax. R package version 1.3.1. https://CRAN.R-project.org/package=kableExtra 


\title{
Supplementary Material
}

Message self and social relevance increases intentions to share content:

Correlational and causal evidence from six studies

\author{
Danielle Cosme \\ Christin Scholz \\ Hang-Yee Chan \\ Bruce P. Doré \\ Prateekshit Pandey \\ José Carreras-Tartak \\ Nicole Cooper \\ Alexandra Paul \\ Shannon M. Burns \\ Emily B. Falk
}

\section{Additional demographic information}

Here, we report available demographic information collapsed across studies. Additional tables summarizing this information for each study separately is available online (https://cnlab.github.io/self-social-sharing/analysis/demographics). In Studies 1, 5, and 6 we also measured socioeconomic status using education and household incoming as indicators. With respect to education, participants reported the following as highest degree completed: $46.3 \%$ Bachelor's degree, 16.1\% some college, 15.8\% Master's degree, 9.9\% Associate's degree, 8.7\% high school graduate, $1.6 \%$ Doctorate degree, 1.4\% Professional school degree, and $0.2 \%$ less than high school. With respect to household income, participants reported the following income brackets: $26.5 \% \$ 50,000$ - \$74,999, $16.8 \% \$ 75,000$ - \$99,999, 16.1\% > \$100,000, 15.1\% $\$ 35,000$ - \$49,999, $10.1 \% \$ 25,000$ - \$34,999, 6.5\% \$16,000 - \$24,999, 3.2\% \$5,000 - \$11,999, $2.8 \% \$ 12,000-\$ 15,999,1.3 \%<\$ 5,000$, and $1.6 \%$ not reported.

\section{Study-specific participant information}

Study 1. In this study, we used existing data from a project investigating the degree to which several message framing interventions might enhance message effectiveness and intentions, norms, and beliefs related to social distancing as a response to the COVID-19 pandemic. This project includes four sub-studies. For the purposes of this paper, the data were collapsed across message framing conditions, since our focus in this paper is on relationships between self and social-relevance and sharing. This study was conducted online through Amazon's Mechanical Turk (MTurk). Participants were included if they were adults 18 or older, residing in the United States, were fluent in English, and passed an initial attention screening question. Participants were excluded based on the standard operating procedures for this project (SOP; https://osf.io/bgs5y/). To be consistent across studies reported in this manuscript, we 
deviated from the project SOP by not trimming outliers to +/- 3 SD. Of the 2470 participants initially recruited, participants were excluded if they failed the English comprehension question $(\mathrm{n}=46)$, the attention screening ( $\mathrm{n}=291)$, knowledge questions about COVID-19 $(\mathrm{n}=14)$, had invariant responses that were more than 3 SDs from the median $(n=13)$, or had more than one of these issues $(\mathrm{n}=29)$. This yielded a final sample of 2081 .

Study 2. This study used existing data from a project examining the effect of several message framing interventions on intentions to vote and perception of norms related to voting. For the purposes of this study, we collapse across message framing conditions, since our focus in this paper is on relationships between self and social-relevance and sharing. The study was conducted online through MTurk. Participants were included if they were adults 18 or older, residing in the United States, were fluent in English, eligible to vote in the U.S. general election, and passed an initial attention screening question. Of the 632 participants initially recruited, participants were excluded if they failed the English comprehension question $(\mathrm{n}=10)$, one or more attention check $(\mathrm{n}=14)$, or had invariant responses that were more than 3 SDs from the median $(n=29$; Med $=22.2 \%, S D=21.3 \%)$, or for more than one of these reasons $(n=32)$. This yielded a final sample of $\mathrm{N}=547$.

Study 3. This study $(\mathrm{N}=248)$ used existing data from a project on civic engagement in college students. The study was conducted online at the University of Pennsylvania. Participants were included if they were adults 18 or older and eligible to vote in the United States. Participants were randomized to one of two message framing conditions, but for the purposes of this paper, the data were collapsed across conditions, since our focus in this paper is on relationships between self and social-relevance and sharing.

Study 4. This study used existing data from a project examining relationships between various message properties and broadcast sharing intentions using headlines from the New York Times. The study was conducted online through MTurk. Participants were included if they were adults 18 or older and were fluent in English. Of the 200 participants who completed the survey, 61 participants were excluded for failing one or more of the English comprehension questions. This yielded a final sample of $N=139$.

Study 5. This preregistered study (https://osf.io/bgs5y/registrations/) was conducted online through MTurk. Participants were included if they were adults 18 or older, residing in the United States, were fluent in English, and passed an initial attention screening question. Participants were excluded based on the standard operating procedures for this project (https://osf.io/bgs5y/). Sample size was based on a power analysis. We determined that with $\mathrm{N}=$ 300 , we would have $>80 \%$ power to detect an effect size of $d=0.05$ for within-person effects and $>95 \%$ power to detect an effect of $d=0.10$ for within- and between-person effects. Of the 408 participants initially recruited, participants were excluded if they failed the English comprehension question $(n=15)$, one or more attention checks $(n=75)$, or the knowledge questions about COVID-19 $(\mathrm{n}=15)$. This yielded a final sample of $\mathrm{N}=315$.

Study 6. This preregistered study (https://osf.io/bgs5y/registrations/) was conducted online through MTurk. The same inclusion and exclusion criteria from Study 5 were used here. 
Sample size was based on a power analysis. We determined that with $N=420$, we would have $>80 \%$ power to detect an effect of $d=0.10$ and $>95 \%$ power to detect an effect of $d=0.15$. Of the 644 participants initially recruited, participants were excluded if they failed the English comprehension question $(n=20)$, one or more attention checks $(n=80)$, or did not provide comprehensible text during the experimental manipulation $(n=233)$. This yielded a final sample of $\mathrm{N}=397$.

\section{Study-specific procedures}

Study 1. Participants were exposed to health messages about social distancing, framed as social media posts on Instagram. In three of the four sub-studies from this project, each participant was exposed to 5 messages drawn randomly from a pool of 15 messages. For the fourth sub-study, each participant saw the same 5 messages. For each message, participants rated self ("This message is relevant to me") and social relevance ("This message is relevant to other people I know"), as well as their intention to share on social media ("I would share this message on social media") using a 7-point scale ( $1=$ strongly disagree, $7=$ strongly agree).

Study 2. Participants were exposed to messages about voting, framed as social media posts for Twitter. Each participant was exposed to 5 messages about voting. For each message, they rated self ("This message is relevant to me") and social relevance ("This message is relevant to people I know"), as well as their intention to share on social media ("I would share this message on social media") using a 100-point scale ( $0=$ strongly disagree, $100=$ strongly agree).

Study 3. Participants were exposed to messages about voting, framed as social media posts for Instagram. Each participant was exposed to 5 messages about voting. For each message, they rated self ("This message is relevant to me") and social relevance ("This message is relevant to people I know"), as well as their broadcast intention to share on social media ("I would share this message on social media") and narrowcast intention to share directly with someone ("I would share this message directly with a friend") using a 100 -point scale $(0=$ strongly disagree, $100=$ strongly agree).

Study 4. Participants were exposed to messages (headline and brief abstract) about health from the New York Times. Each participant was exposed to 8 messages randomly drawn from a pool of 80 articles. For each message, they rated self ("How relevant is this content to you?") and social relevance ("How relevant is this content to other people?"), as well as their sharing intention ("How much would you want to share this article with other people?") using a 10 -point scale $(0=$ not at all, $10=$ very much $)$.

Study 5. Participants were exposed to messages (headline and brief abstract) about COVID-19 or climate change from the New York Times. Each participant was exposed to 10 messages, 5 about COVID-19 and 5 about climate change. Each participant was randomly assigned to one of 11 stimuli sets that included articles matched for popularity. For each message, they rated self ("This message is relevant to me") and social relevance ("This message is relevant to people I know") using a 100-point scale ( $0=$ strongly disagree, $100=$ strongly agree), as well as their broadcast intention to share on social media ("How much do you want to 
share this article by posting on your social media (on Facebook, Twitter, etc)?") and narrowcast intention to share directly with someone ("How much do you want to share this article directly with someone you know (via email, direct message, etc)?") using a 100-point scale ( 0 = not at all, 100 = very much).

Study 6. Participants saw the following instructions at the beginning of the study:

In this study, we are interested in understanding how different people react to online news, and in what ways information is shared among people. You will review a set of 10 actual news headlines taken from the internet. We would like you to read the article headlines, write short comments and give ratings. This writing task will help you reflect upon the headlines in different ways.

In order to help you reflect on these articles in different ways, we'd like you to write short comments to accompany each article, as if you were sharing on social media. In particular, we will ask you to write comments for each article with one of these specific goals in mind:

Write a short comment to describe why this article matters to you personally. Write a short comment to describe why this article matters to people you know. Write a short comment to describe what this article is about.

Messages consisted of a news headline and brief abstract from the New York Times about general health or climate change. These messages were sampled from a pool of 55 articles per topic and each participant was randomized to one of 11 sets of articles that contained 5 messages about health and 5 about climate change, matched with respect to the web traffic the news article has generated (specifically, the number of click-throughs for the article URL). For each message, participants wrote a comment based on the experimental condition and rated self ("This message is relevant to me") and social relevance ("This message is relevant to people I know") using a 100-point scale ( $0=$ strongly disagree, $100=$ strongly agree $)$, as well as their broadcast intention to share on social media ("I would share this article by posting on social media (on Facebook, Twitter, etc)") and narrowcast intention to share directly with someone ("I would share this article directly with someone I know (via email, direct message, etc)") using a 100 -point scale $(0=$ strongly disagree, $100=$ strongly agree $)$.

\section{Software packages}

All models were estimated using the lme4 (Version 1.1-26; Bates et al., 2015) and lmerTest (Version 3.1-3; Kuznetsova, Brockhoff, \& Christensen, 2017) for significance testing in $\mathrm{R}$ (Version 3.6.3; R Core Team, 2020). The specification curve analysis was implemented using code adapted from specr (Masur \& Scharkow, 2020). The Bayesian mediation analyses were conducted using brms (Version 2.16.3; Bürkner, 2017) and visualized using tidybayes (Version 3.0.2; Kay, 2022). Additional software packages used to conduct these analyses in R include: 
boot (Version 1.3-24; Canty \& Ripley, 2019), broom.mixed (Version 0.2.7; Bolker \& Robinson, 2021), dplyr (Version 1.0.7; Wickham et al., 2021), forcats (Version 0.5.1; Wickham, 2021), EMAtools (Version 0.1.4; Kleiman, 2021) furrr (Version 0.2.2; Vaughn \& Dancho, 2021), ggplot2 (Version 3.3.5; Wickham, 2019), ggpubr (Version 0.4.0; Kassambara, 2020), kableExtra (Version 1.3.1; Zhu, 2020), knitr (Version 1.31; Xie, 2021), Matrix (Version 1.2-18; Bates \& Maechler, 2019), purrr (Version 0.3.4; Henry \& Wickham, 2020), rmcorr (Version 0.4.5; Bakdash \& Marusich, 2017), readr (Version 1.4.0; Wickham \& Hester, 2020), report (Version 0.3.5; Makowski et al., 2020), stringr (Version 1.4.0; Wickham, 2019), tibble (Version 3.1.2; Müller \& Wickham, 2021), tidyr (Version 1.1.3; Wickham, 2021), tidytext (Version 0.3.2; Silge \& Robinson, 2016), and tidyverse (Wickhman, 2019).

\section{Mega-analysis with downsampled data}

Message-level correlations between self and social relevance. First, we conducted exploratory analyses looking at the correlation between self and social relevance for each message in each study. These correlations are visualized in Figure S1, and the average correlation strength and variability for each study and message content domain are reported in Table S1. The messages about climate change were used in both Study 5 and 6, but are treated separately for each study.

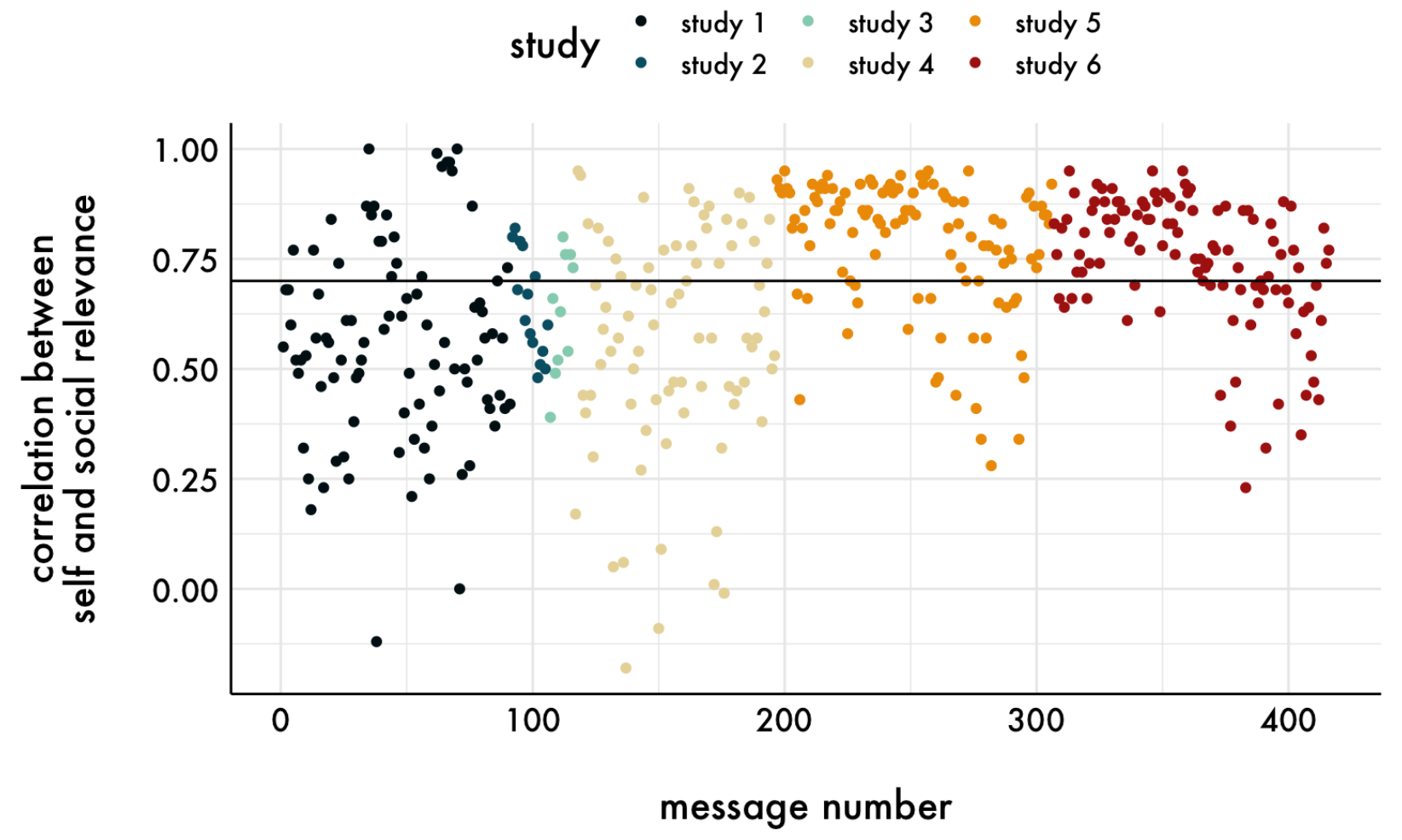

Figure S1. Message-level correlations between self and social relevance as a function of study. The horizontal line is $r=.70$ and is the cutoff used in the downsampled mega-analysis. 
SHARING, SELF AND SOCIAL RELEVANCE

Table S1

Descriptive statistics about message-level correlations between self and social relevance as a function of study and content domain

\begin{tabular}{lcccc}
\hline Study & Content & Correlation $M$ & Correlation $S D$ & Correlation Range \\
\hline Study 1 & COVID-19 & 0.56 & 0.23 & $-0.12,1.00$ \\
Study 2 & Voting & 0.64 & 0.12 & $0.48,0.82$ \\
Study 3 & Voting & 0.63 & 0.14 & $0.39,0.80$ \\
Study 4 & Health & 0.56 & 0.26 & $-0.18,0.95$ \\
Study 5 & Climate & 0.84 & 0.11 & $0.43,0.95$ \\
& COVID-19 & 0.73 & 0.17 & $0.28,0.95$ \\
Study 6 & Climate & 0.82 & 0.09 & $0.61,0.95$ \\
& Health & 0.67 & 0.16 & $0.23,0.88$ \\
\hline
\end{tabular}

Downsampled mega-analysis. Although the variance inflation factors (VIF) for the variables included in the mega-analysis reported in the main manuscript were small to moderate $($ VIF range $=1.00-4.24$ ), we conducted a sensitivity analysis to assess the impact of multicollinearity on the model. Specifically, we estimated the same mega-analysis model reported in the main manuscript in a subset of the data that had message-level correlations below $r=.70$. This threshold for downsampling was selected as a benchmark because it means that half $(49 \%)$ of the variance is shared between variables.

These results are consistent with those reported in the main manuscript (Figure S2; Table $\mathrm{S} 2$ ). All parameter estimates were in the same direction and did not deviate substantially with respect to magnitude from those in the original model (deviation range $=0.00-0.04)$. The largest deviation was for the interaction between sharing type and between-person self-relevance, such that the difference between broadcasting and narrowcasting decreased. 


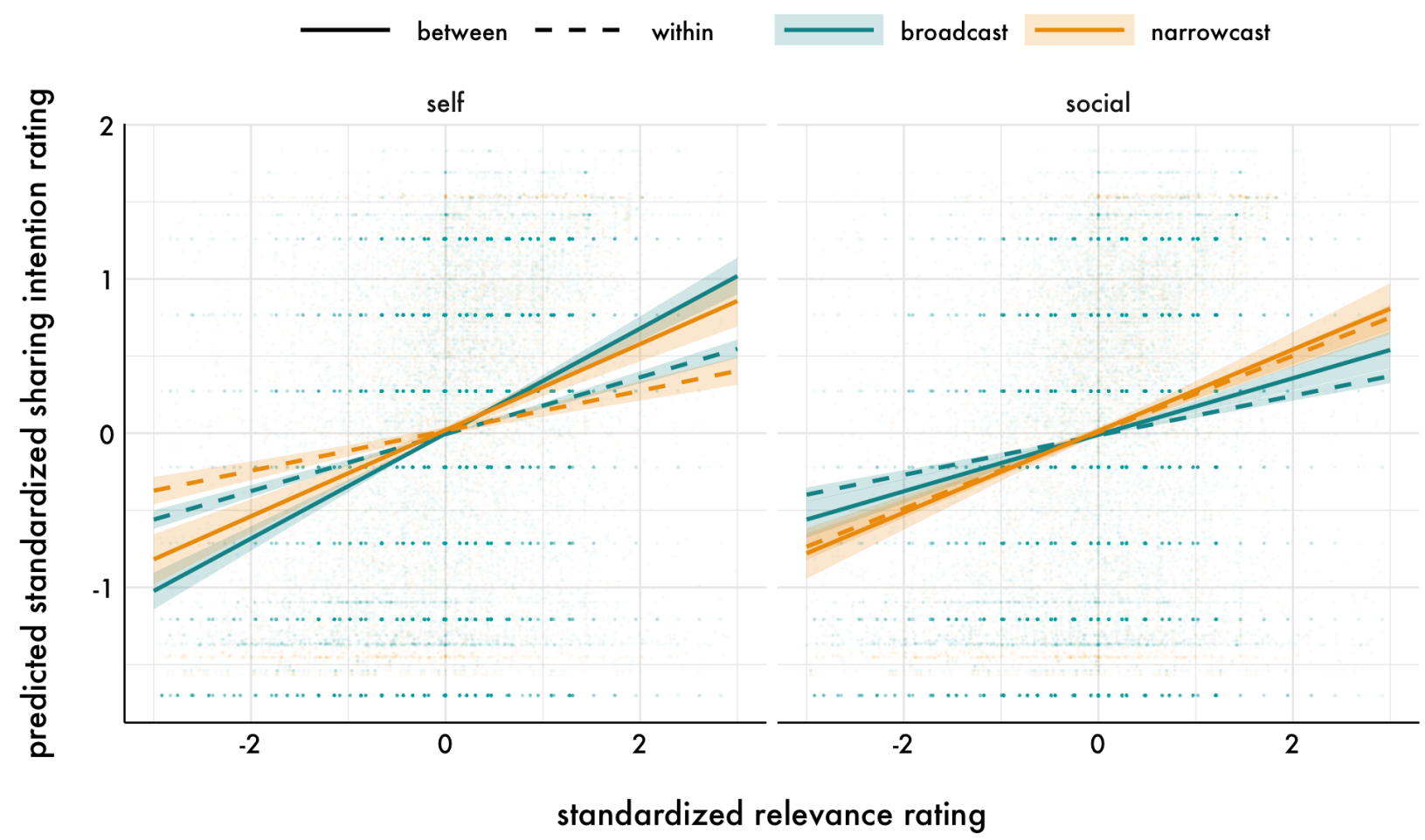

Figure S2. The predicted within- and between-person relationships for relevance ratings and sharing intention ratings from the mega-analysis as a function of within- and between-person relevance variable (self or social) and sharing type (broad- or narrowcasting) estimated from the downsampled data. The points represent the raw message-level responses; error bands are $95 \%$ confidence intervals. The left panel visualizes the relationships between sharing intentions and self-relevance, and shows that the relationship with sharing intentions is stronger when broadcasting compared to narrowcasting for both within- and between-person self-relevance. The right panel visualizes the relationships between sharing intentions and social relevance, and shows that the relationship with sharing intentions is stronger when narrowcasting compared to broadcasting for within- and between-person social relevance.

Table S2

Results from the downsampled mega-analysis model

\begin{tabular}{lcccc}
\hline Parameter & $\beta[95 \% \mathrm{CI}]$ & $d f$ & $t$ & $p$ \\
\hline Sharing type & $0.02[-0.00,0.04]$ & 12764.56 & 1.54 & .120 \\
Self between & $0.34[0.30,0.38]$ & 3683.12 & 16.80 & $<.001$ \\
Self within & $0.18[0.16,0.20]$ & 136.37 & 18.01 & $<.001$ \\
Social between & $0.18[0.14,0.22]$ & 3653.76 & 9.08 & $<.001$ \\
Social within & $0.13[0.11,0.14]$ & 77.55 & 16.08 & $<.001$ \\
Self between x Sharing type & $-0.06[-0.10,-0.02]$ & 12786.35 & 2.87 & $<.001$ \\
Self within x Sharing type & $-0.06[-0.08,-0.03]$ & 5772.64 & 4.17 & $<.001$ \\
Social between x Sharing type & $0.08[0.04,0.12]$ & 12780.84 & 3.77 & $<.001$ \\
Social within x Sharing type & $0.12[0.09,0.15]$ & 3203.99 & 8.80 & $<.001$ \\
\hline
\end{tabular}

Note. "Within" parameters refer to the person-centered level-1 predictors, whereas "between" parameters refer to grand-mean centered level-2 predictors. The reference group for sharing type is broadcast sharing intentions. Coefficients are in standardized units. Degrees of freedom $(d f)$ were calculated using the Satterthwaite approximation. 


\section{Mega-analysis estimated separately for self and social relevance}

Although the down-sampled analyses replicate the results in the main manuscript and suggest that the findings hold when self and social relevance are less strongly correlated, we also estimated post-hoc mega-analyses for self and social relevance separately (Table S3; Figure S3). These results are consistent with the results reported from the mega-analysis estimating the unique associations with sharing intentions reported in the main manuscript with the exception that within- and between-person self-relevance were no longer more strongly associated with broadcasting compared to narrowcasting.

Table S3

Results from the separated mega-analysis models

\begin{tabular}{lcccc}
\hline Self-relevance & \multicolumn{4}{c}{$t$} \\
\hline Parameter & $\beta[95 \% \mathrm{CI}]$ & $d f$ & $t$ & $p$ \\
\hline Sharing type & $-0.00[-0.01,0.01]$ & 25634.78 & 0.02 & .990 \\
Self between & $0.50[0.47,0.52]$ & 3835.90 & 44.57 & $<.001$ \\
Self within & $0.26[0.24,0.28]$ & 428.70 & 32.55 & $<.001$ \\
Self between x Sharing type & $-0.01[-0.02,0.00]$ & 25347.91 & 1.31 & .190 \\
Self within x Sharing type & $0.01[-0.00,0.03]$ & 22988.68 & 1.91 & .060 \\
& & & & \\
Social relevance & $\beta[95 \% \mathrm{CI}]$ & $d f$ & $t$ & $p$ \\
\hline Parameter & $-0.00[-0.01,0.01]$ & 25349.81 & 0.05 & .960 \\
Sharing type & $0.46[0.44,0.48]$ & 3812.97 & 40.34 & $<.001$ \\
Social between & $0.24[0.23,0.26]$ & 409.77 & 32.52 & $<.001$ \\
Social within & $0.02[0.00,0.03]$ & 25050.87 & 2.28 & .020 \\
Social between x Sharing type & $0.07[0.06,0.08]$ & 22318.42 & 9.59 & $<.001$ \\
Social within x Sharing type & & & \\
\hline
\end{tabular}

Note. "Within" parameters refer to the person-centered level-1 predictors, whereas "between" parameters refer to grand-mean centered level-2 predictors. The reference group for sharing type is broadcast sharing intentions. Coefficients are in standardized units. Degrees of freedom $(d f)$ were calculated using the Satterthwaite approximation. 


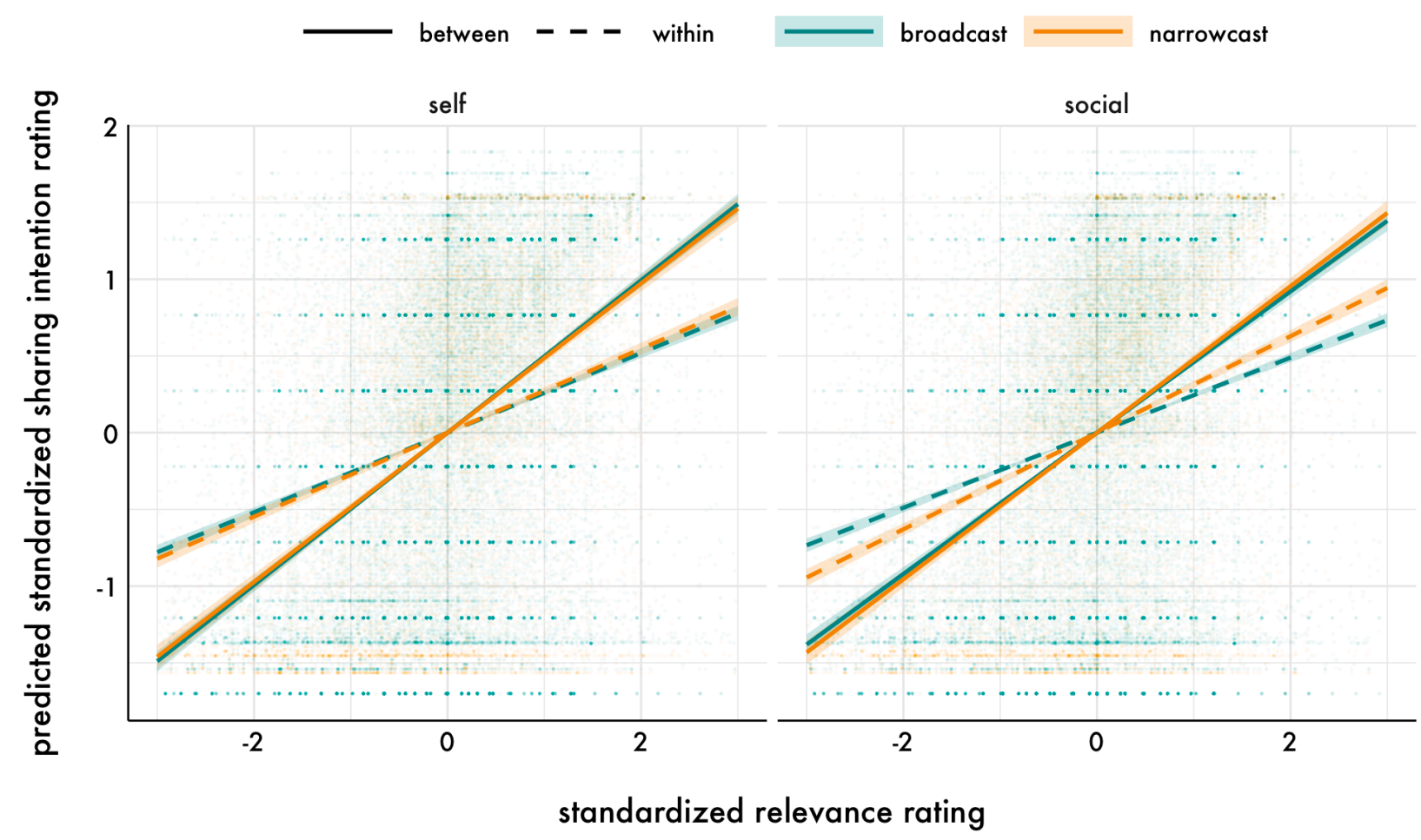

Figure S3. The predicted within- and between-person relationships for relevance ratings and sharing intention ratings from the mega-analyses estimated for self and social relevance separately, as a function of within- and between-person relevance variable (self or social) and sharing type (broad- or narrowcasting) estimated from the downsampled data. The points represent the raw message-level responses; error bands are 95\% confidence intervals. The left panel visualizes the relationships between sharing intentions and self-relevance, and shows that the relationship with sharing intentions is equivalent when broadcasting and narrowcasting for both within- and between-person self-relevance. The right panel visualizes the relationships between sharing intentions and social relevance, and shows that the relationship with sharing intentions is stronger when narrowcasting compared to broadcasting for withinand between-person social relevance.

\section{Additional information about the specification curve analysis}

As described in the main manuscript, the specification curve analysis explores the robustness of the relationships between self and social relevance and sharing intentions to inclusion of covariates and across different subsets of the data. Table S4 describes the 13 subsets that were included in the analysis. Figures S4-5 depict the curve for each relevance variable including a marker for which subset the model was estimated in. Descriptive statistics for the curve for each relevance variable separately is reported in Table S5 as a function of sharing type and message medium. Figure S5-6 includes all relevance variables in the same specification curve in order to compare them (versus showing the curve for each relevance variable separately in the main manuscript). 
SHARING, SELF AND SOCIAL RELEVANCE

Table S4

Data subsets included in the specification curve analysis

\begin{tabular}{lccccc}
\hline Subset & Content & Medium & Sharing type Studies & $\begin{array}{c}\text { N } \\
\text { models }\end{array}$ \\
\hline 1 & COVID-19 & Social media & Broadcast & 1 & 28 \\
2 & COVID-19 & Newspapers & Broadcast & 5 & 28 \\
3 & COVID-19 & Newspapers & Narrowcast & 5 & 28 \\
4 & Voting & Social media & Broadcast & $2 \& 3$ & 20 \\
5 & Voting & Social media & Narrowcast & 3 & 16 \\
6 & Health & Newspapers & Broadcast & $4 \& 6$ & 28 \\
7 & Health & Newspapers & Narrowcast & 6 & 28 \\
8 & Climate change & Newspapers & Broadcast & $5 \& 6$ & 28 \\
9 & Climate change & Newspapers & Narrowcast & $5 \& 6$ & 28 \\
10 & COVID-19 & Social media \& & Broadcast & $1 \& 5$ & 28 \\
11 & COVID-19 \& voting & newspapers & Broadcast & $1,2 \& 3$ & 28 \\
12 & COVID-19, health \& climate & Newspapers & Broadcast & $4,5 \& 6$ & 28 \\
13 & COVID-19 \& climate change & Newspapers & Narrowcast & $5 \& 6$ & 28 \\
\hline
\end{tabular}


SHARING, SELF AND SOCIAL RELEVANCE
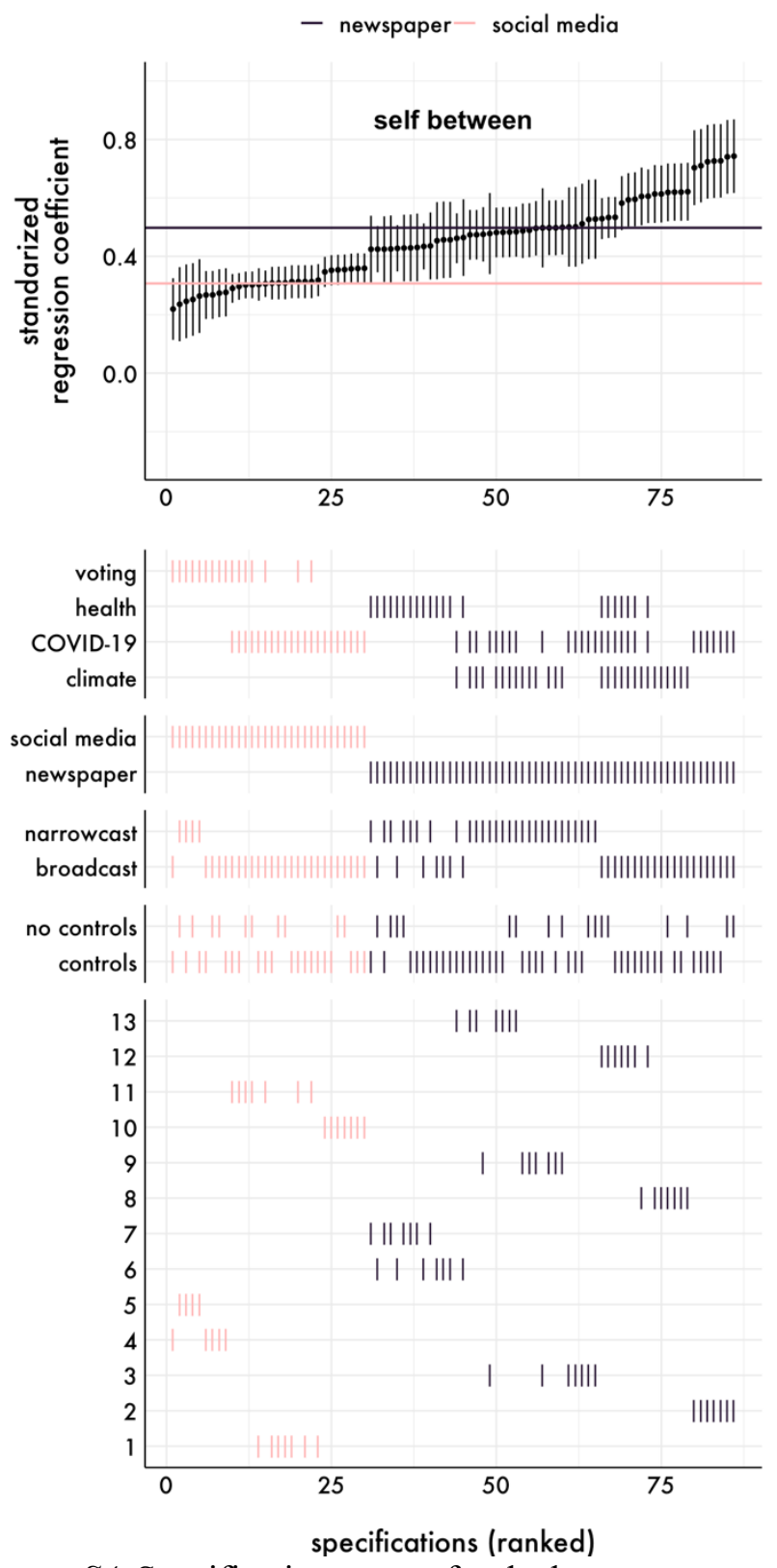
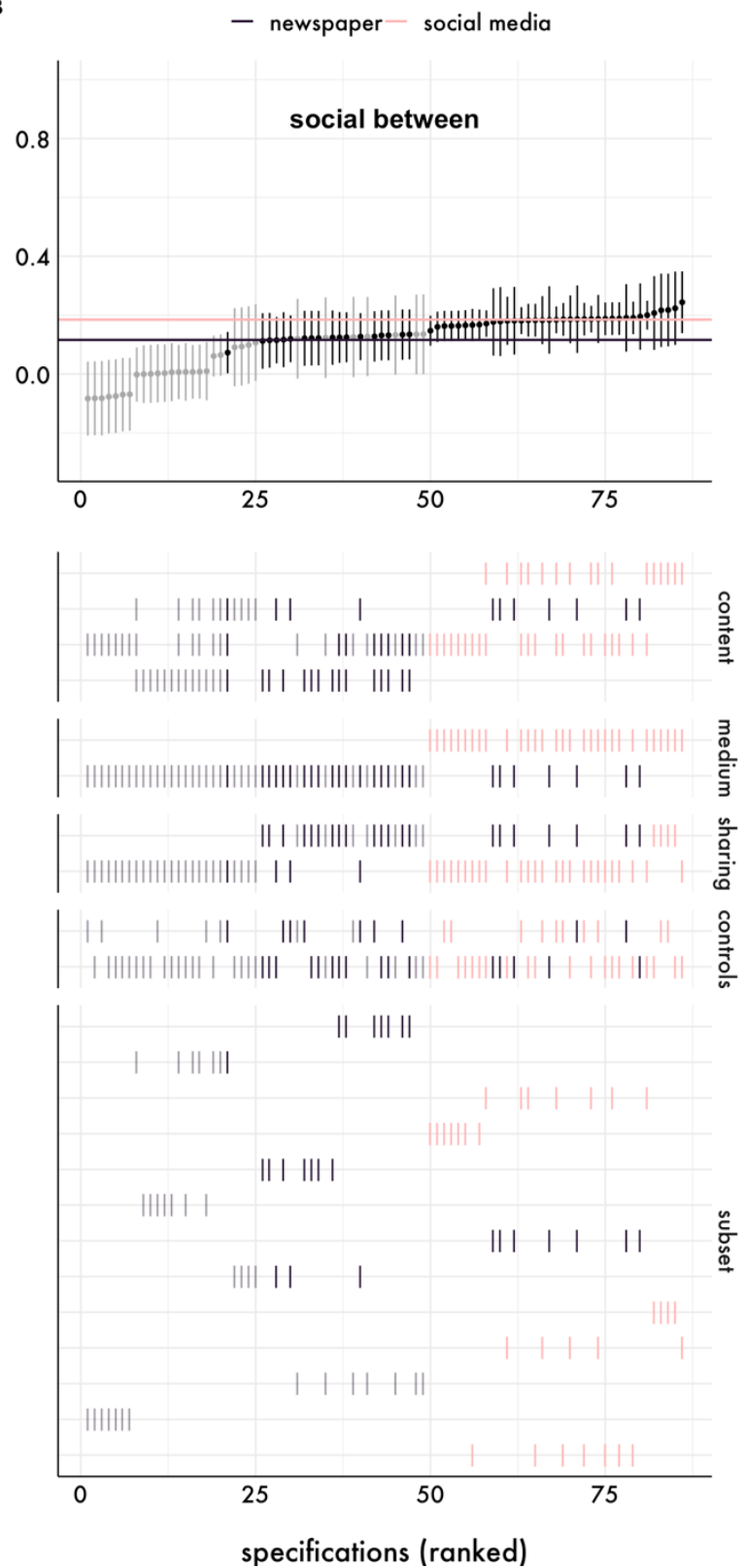

Figure S4. Specification curves for the between-person relevance variables reported in Figure 3A-B including an additional marker for which subset the model was estimated in. The subsets are described in Table S4. 
SHARING, SELF AND SOCIAL RELEVANCE

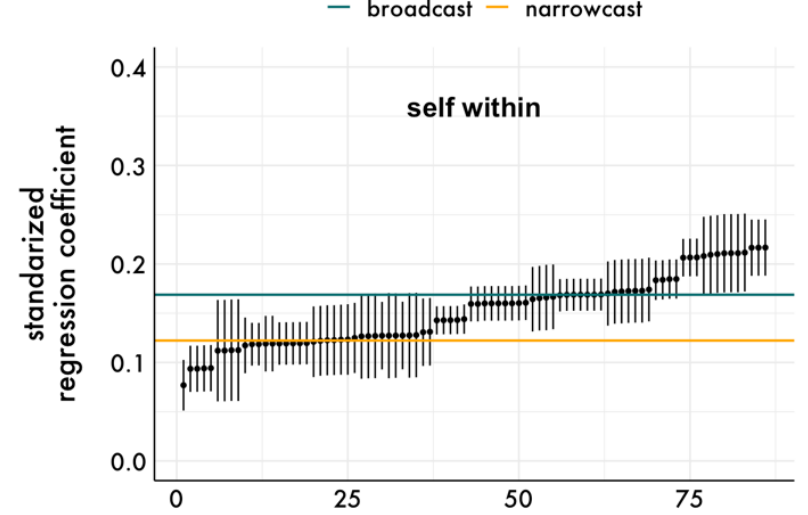

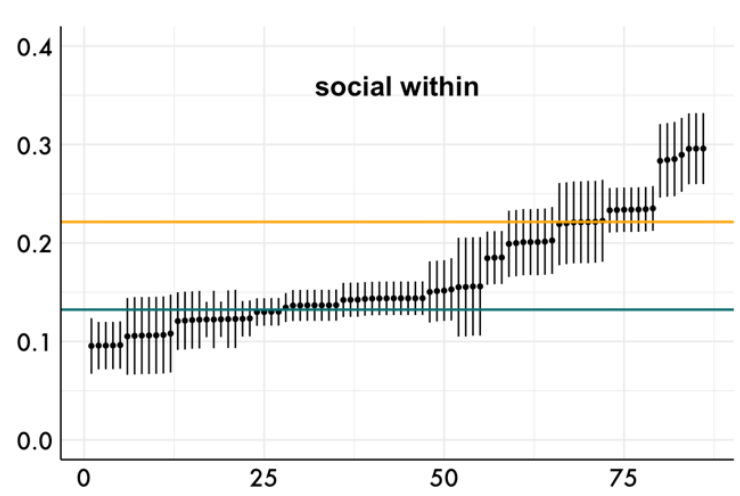
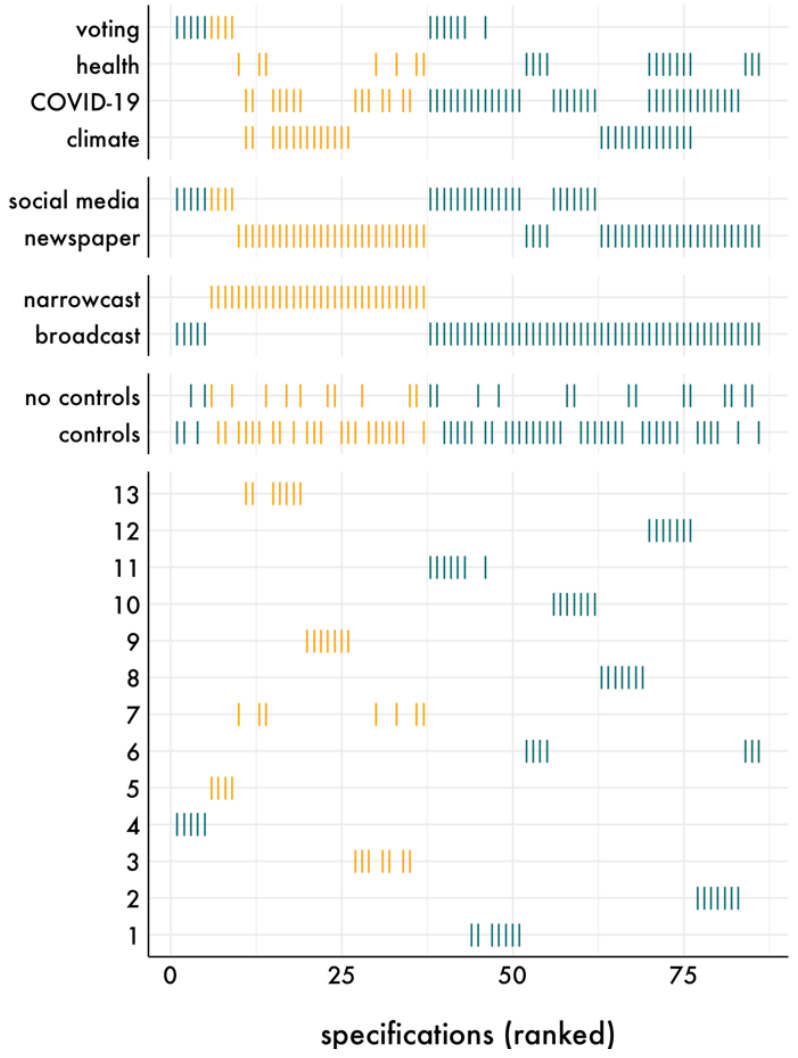

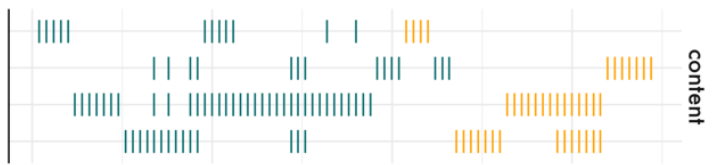

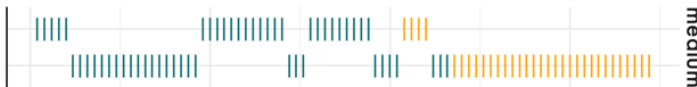

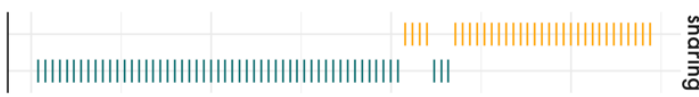

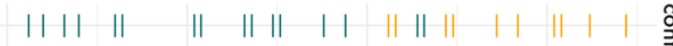
|| ||| | |||| ||||||||| ||||| || ||||| || ||||| || || ||||||| ||| ||| ||| $\frac{\overrightarrow{3}}{n}$

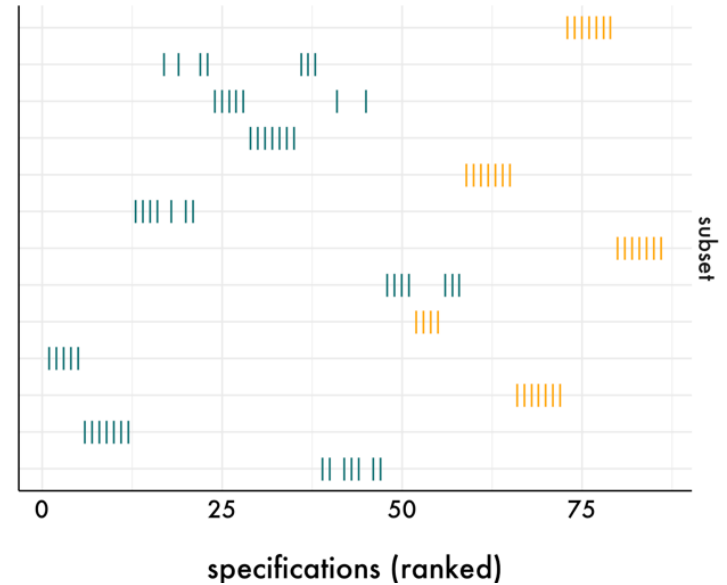

Figure S5. Specification curves for the within-person relevance variables reported in Figure 3C-D including an additional marker for which subset the model was estimated in. The subsets are described in Table S4. 
SHARING, SELF AND SOCIAL RELEVANCE

Table S5

Specification curve descriptives statistics by sharing type and message medium

\begin{tabular}{|c|c|c|c|c|c|}
\hline \multicolumn{6}{|l|}{ Sharing type } \\
\hline Parameter & Grouping variable & Median $\beta$ & $\beta \quad \beta$ Range & Positive \& significant & Negative \& significant \\
\hline \multirow[t]{2}{*}{ Self between } & Broadcast & 0.43 & $0.22,0.74$ & $100.00 \%$ & $0.00 \%$ \\
\hline & Narrowcast & 0.48 & $0.24,0.53$ & $100.00 \%$ & $0.00 \%$ \\
\hline \multirow[t]{2}{*}{ Self within } & Broadcast & 0.17 & $0.08,0.22$ & $100.00 \%$ & $0.00 \%$ \\
\hline & Narrowcast & 0.12 & $0.11,0.13$ & $100.00 \%$ & $0.00 \%$ \\
\hline \multirow[t]{2}{*}{ Social between } & Broadcast & 0.12 & $-0.08,0.24$ & $55.56 \%$ & $0.00 \%$ \\
\hline & Narrowcast & 0.13 & $0.11,0.22$ & $78.12 \%$ & $0.00 \%$ \\
\hline \multirow[t]{2}{*}{ Social within } & Broadcast & 0.13 & $0.10,0.19$ & $100.00 \%$ & $0.00 \%$ \\
\hline & Narrowcast & 0.22 & $0.16,0.30$ & $100.00 \%$ & $0.00 \%$ \\
\hline \multicolumn{6}{|l|}{ Message medium } \\
\hline Parameter & Grouping variable & Median $\beta$ & $3 \beta$ Range & Positive \& significant & Negative \& significant \\
\hline \multirow[t]{2}{*}{ Self between } & Newspaper & 0.50 & $0.42,0.74$ & $100.00 \%$ & $0.00 \%$ \\
\hline & Social media & 0.31 & $0.22,0.36$ & $100.00 \%$ & $0.00 \%$ \\
\hline \multirow{2}{*}{ Self within } & Newspaper & 0.15 & $0.12,0.22$ & $100.00 \%$ & $0.00 \%$ \\
\hline & Social media & 0.16 & $0.08,0.17$ & $100.00 \%$ & $0.00 \%$ \\
\hline \multirow[t]{2}{*}{ Social between } & Newspaper & 0.12 & $-0.08,0.20$ & $44.64 \%$ & $0.00 \%$ \\
\hline & Social media & 0.18 & $0.15,0.24$ & $100.00 \%$ & $0.00 \%$ \\
\hline \multirow{2}{*}{ Social within } & Newspaper & 0.19 & $0.11,0.30$ & $100.00 \%$ & $0.00 \%$ \\
\hline & Social media & 0.14 & $0.10,0.16$ & $100.00 \%$ & $0.00 \%$ \\
\hline
\end{tabular}


SHARING, SELF AND SOCIAL RELEVANCE

A

- self between - self within $\quad-$ social between - social within

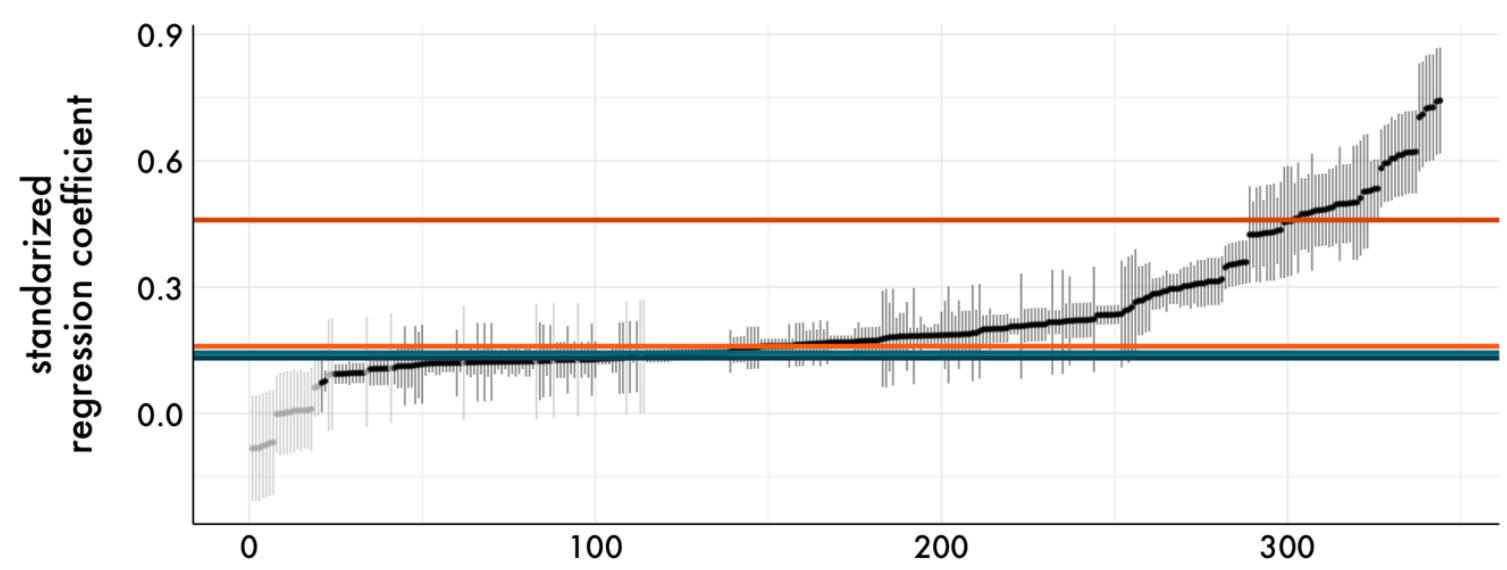

B

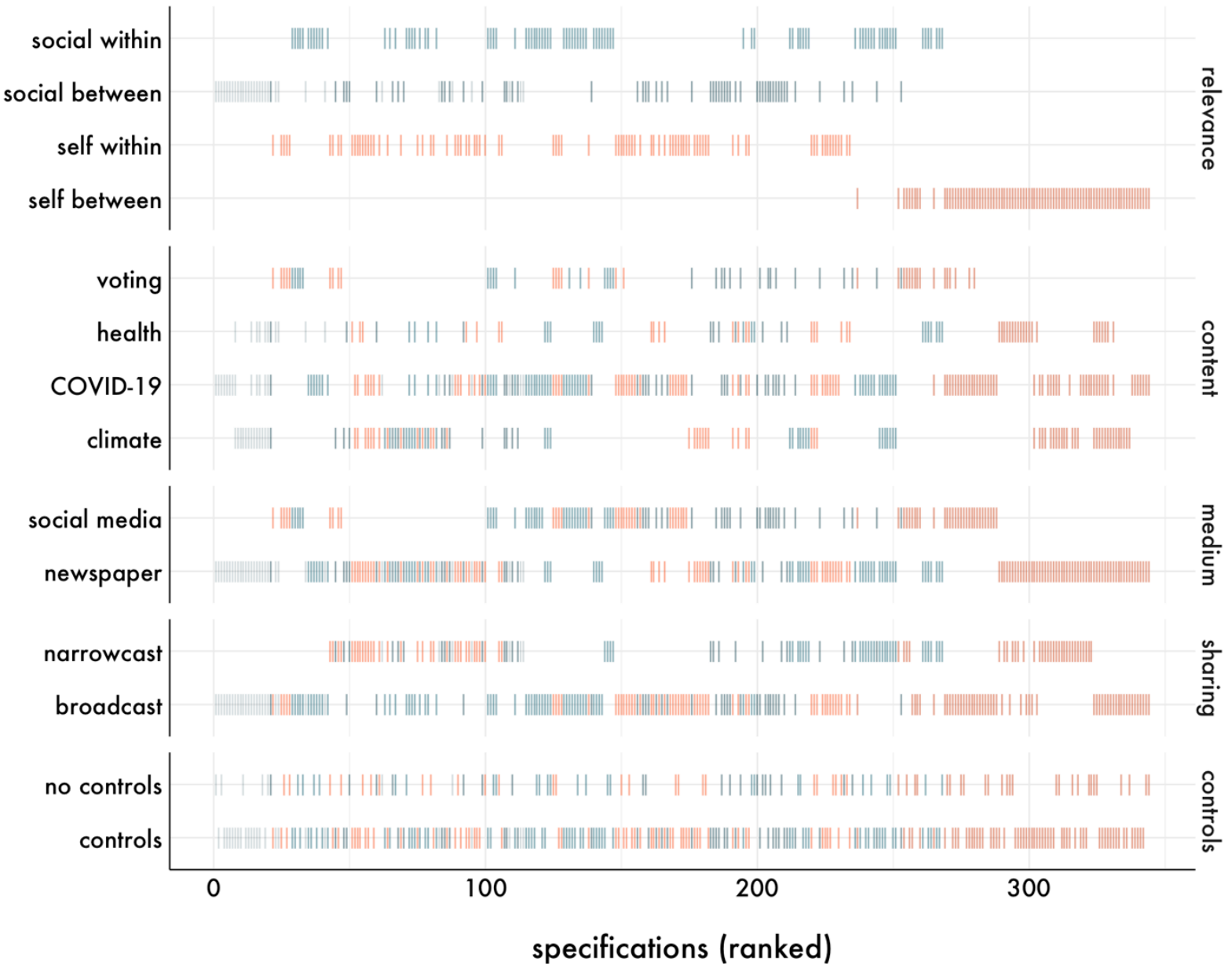

Figure S6. Specification curve visualizing the relationship between self and social relevance and sharing intentions across analytic decisions and subsets of the data. (A) The top panel depicts the relationship between the relevance variables and sharing intentions. Each dot represents the standardized regression coefficient for the relevance variable of interest from a unique model specification with a $95 \%$ confidence interval around it. Model specifications are ordered by the regression coefficient; models for which the regression coefficient of interest was statistically significant at $p<.05$ are visualized in black, whereas 
coefficients $p>.05$ are in gray. The colored horizontal lines represent the median regression coefficient across model specifications for each relevance variable, separately. (B) The bottom panel shows the relevance variables and analytic decisions that were included in each model specification. Model specifications are colored based on the relevance variable; models for which the regression coefficient of interest was statistically significant at $p<0.05$ are visualized are opaque, whereas coefficients $p>0.05$ are partially opaque. Content $=$ content type; medium $=$ message medium; type $=$ sharing type; controls $=$ inclusion of demographic covariates.

\section{Results from analyses estimated separately for each study}

For completeness and to be consistent with our preregistered analysis plans for Studies 5 and 6, we also report the results for each study separately. As in the mega-analysis reported in the main manuscript, we investigated the relationships between message self and social relevance and broadcast sharing intentions using multilevel modeling. Self and social relevance ratings were disaggregated into within and between-person variables. The "within-person" self and social relevance variables were level 1 predictors, centered within-person (i.e., "centered within context") and standardized across people. Each of the "between-person" variables were level 2 predictors created by averaging across message self or social relevance ratings to create a single average per person that was then grand-mean centered and standardized across people.

For each study, we estimated three multilevel models regressing message sharing intentions on 1) within- and between-person self-relevance, 2) within- and between-person social relevance, and 3) within- and between-person self-relevance, and within- and between-person social relevance. The first and second models estimate the relationship between sharing intentions and self and social relevance separately, whereas the third model estimates each variables' unique association with sharing intentions after adjusting for the others. In all models, intercepts and within-person relevance variables were allowed to vary randomly across people and intercepts could vary across messages. This was the least constrained random effects structure that converged across studies. All models were estimated using the lme4 (Version 1.126; Bates et al., 2015) and lmerTest (Version 3.1-3; Kuznetsova, Brockhoff, \& Christensen, 2017) for significance testing in $\mathrm{R}$ (Version 3.6.3; R Core Team, 2020).

For the studies that included broad- and narrowcasting, we examined potential differences between broadcast and narrowcast sharing intentions by estimating a fourth model that included sharing type (broadcast or narrowcast) as a moderator of the relationship between self or social relevance and sharing intentions. In these models, intercepts and within-person relevance variables were allowed to vary randomly across people and messages, which was the least constrained random effects structure that converged across studies. 


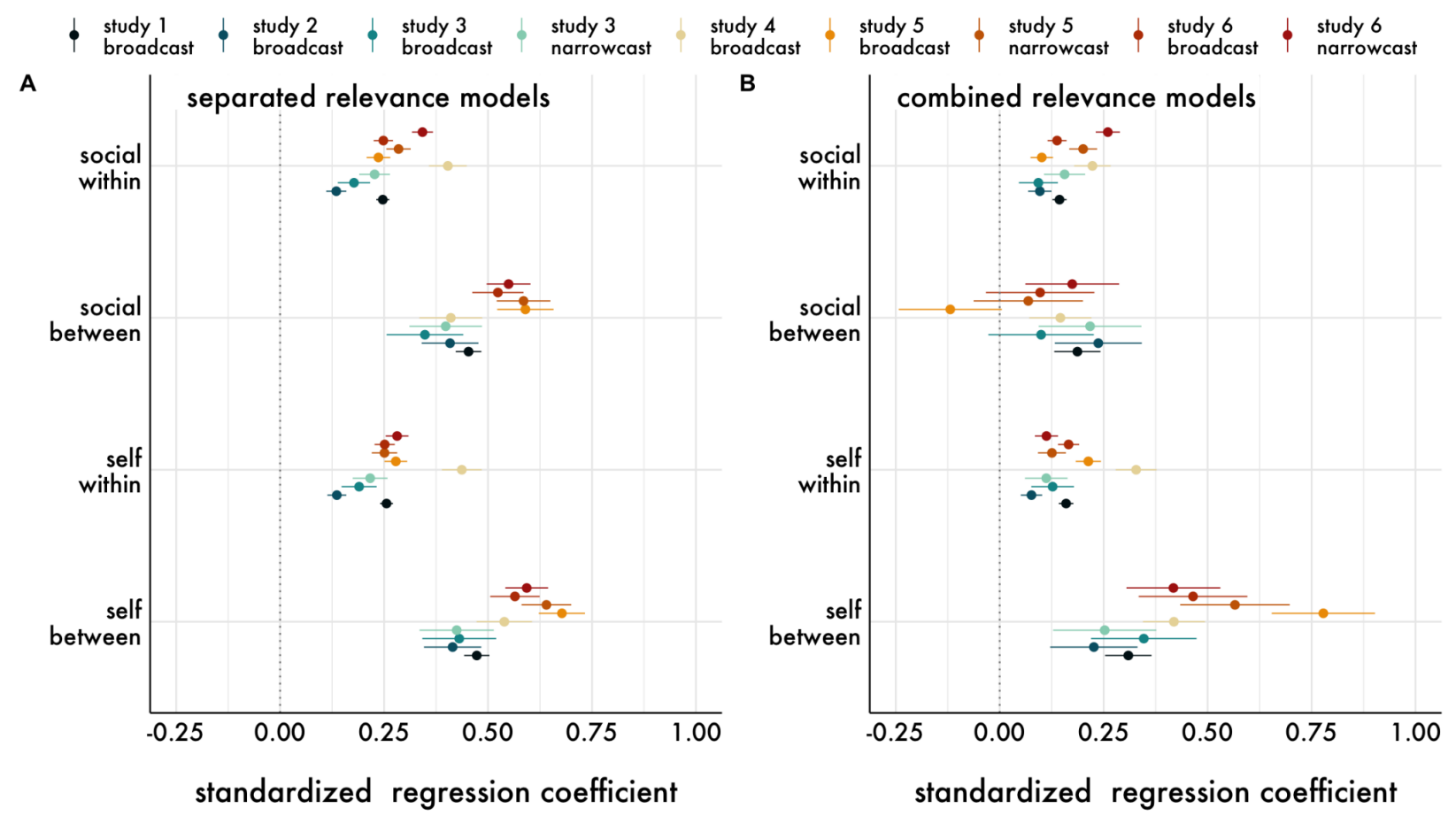

Figure S7. Standardized regression coefficients from (A) the models run separately including either the self-relevance or social relevance variables only, and (B) the models including the self and social relevance variables within the same model. "Within" parameters refer to the person-centered level 1 predictors, whereas "between" parameters refer to grand-mean centered level 2 predictors. Error bars around the point estimates are $95 \%$ confidence intervals.

First, we estimated the association between each relevance variable and sharing intention separately (Figure S7A; Tables S6-7). In all studies, within- and between-person self and social relevance were positively related to broad- and narrowcast sharing intentions, and the magnitude ranged from small to large effects.

Table S6

Results from the self-relevance multilevel models

\begin{tabular}{lccccc}
\hline Model & Parameter & $\beta[95 \% \mathrm{CI}]$ & $d f$ & $t$ & $p$ \\
\hline Study 1 broadcast & Self between & $0.47[0.44,0.50]$ & 2145.50 & 30.47 & $<.001$ \\
& Self within & $0.26[0.24,0.27]$ & 1181.80 & 32.32 & $<.001$ \\
Study 2 broadcast & Self between & $0.41[0.35,0.48]$ & 555.65 & 11.81 & $<.001$ \\
& Self within & $0.14[0.11,0.16]$ & 265.79 & 11.74 & $<.001$ \\
Study 3 broadcast & Self between & $0.43[0.34,0.52]$ & 247.14 & 9.51 & $<.001$ \\
& Self within & $0.19[0.15,0.23]$ & 158.23 & 8.82 & $<.001$ \\
Study 3 narrowcast & Self between & $0.42[0.34,0.51]$ & 249.16 & 9.32 & $<.001$ \\
& Self within & $0.22[0.17,0.26]$ & 161.08 & 10.14 & $<.001$ \\
Study 4 broadcast & Self between & $0.54[0.47,0.61]$ & 142.31 & 15.88 & $<.001$ \\
& Self within & $0.44[0.39,0.48]$ & 121.19 & 17.93 & $<.001$ \\
Study 5 broadcast & Self between & $0.68[0.62,0.73]$ & 324.22 & 23.96 & $<.001$ \\
& Self within & $0.28[0.25,0.31]$ & 245.44 & 19.97 & $<.001$
\end{tabular}




\begin{tabular}{lccccc} 
Study 5 narrowcast & Self between & $0.64[0.58,0.70]$ & 317.64 & 21.03 & $<.001$ \\
& Self within & $0.25[0.22,0.28]$ & 229.36 & 16.06 & $<.001$ \\
Study 6 broadcast & Self between & $0.56[0.51,0.62]$ & 405.99 & 18.60 & $<.001$ \\
& Self within & $0.25[0.23,0.28]$ & 334.94 & 20.35 & $<.001$ \\
Study 6 narrowcast & Self between & $0.59[0.54,0.64]$ & 398.73 & 22.62 & $<.001$ \\
& Self within & $0.28[0.25,0.31]$ & 328.95 & 20.12 & $<.001$ \\
\hline
\end{tabular}

Note. "Within" parameters refer to the person-centered level 1 predictors, whereas "between" parameters refer to grand-mean centered level 2 predictors. Coefficients are in standardized units. Degrees of freedom $(d f)$ were calculated using the Satterthwaite approximation.

Table S7

Results from the social relevance multilevel models

\begin{tabular}{lccccc}
\hline Model & Parameter & $\beta[95 \% \mathrm{CI}]$ & $d f$ & $t$ & $p$ \\
\hline Study 1 broadcast & Social between & $0.45[0.42,0.48]$ & 2133.49 & 28.84 & $<.001$ \\
& Social within & $0.25[0.23,0.26]$ & 967.20 & 30.88 & $<.001$ \\
Study 2 broadcast & Social between & $0.41[0.34,0.48]$ & 548.93 & 11.74 & $<.001$ \\
& Social within & $0.14[0.11,0.16]$ & 272.42 & 10.95 & $<.001$ \\
Study 3 broadcast & Social between & $0.35[0.26,0.44]$ & 239.36 & 7.41 & $<.001$ \\
& Social within & $0.18[0.14,0.22]$ & 128.06 & 8.95 & $<.001$ \\
Study 3 narrowcast & Social between & $0.40[0.31,0.49]$ & 234.77 & 8.95 & $<.001$ \\
& Social within & $0.23[0.19,0.26]$ & 111.96 & 12.07 & $<.001$ \\
Study 4 broadcast & Social between & $0.41[0.33,0.49]$ & 139.34 & 10.66 & $<.001$ \\
& Social within & $0.40[0.36,0.45]$ & 103.95 & 17.47 & $<.001$ \\
Study 5 broadcast & Social between & $0.59[0.52,0.66]$ & 316.18 & 17.05 & $<.001$ \\
& Social within & $0.24[0.21,0.26]$ & 224.97 & 16.29 & $<.001$ \\
Study 5 narrowcast & Social between & $0.59[0.52,0.65]$ & 317.74 & 17.76 & $<.001$ \\
& Social within & $0.28[0.26,0.31]$ & 225.47 & 19.10 & $<.001$ \\
Study 6 broadcast & Social between & $0.52[0.46,0.59]$ & 402.78 & 16.68 & $<.001$ \\
& Social within & $0.25[0.22,0.27]$ & 303.88 & 20.92 & $<.001$ \\
Study 6 narrowcast & Social between & $0.55[0.50,0.60]$ & 401.49 & 20.42 & $<.001$ \\
& Social within & $0.34[0.32,0.37]$ & 287.96 & 26.55 & $<.001$ \\
\hline
\end{tabular}

Note. "Within" parameters refer to the person-centered level 1 predictors, whereas "between" parameters refer to grand-mean centered level 2 predictors. Coefficients are in standardized units. Degrees of freedom $(d f)$ were calculated using the Satterthwaite approximation.

Next, we tested whether self and social relevance accounted for unique variance when estimated within the same model, meaning that parameter estimates reflect the relationship after adjusting for the other variables in the model (Figure S7B; Table S8). All relationships between within- and between-person self and social relevance and sharing intentions were positive except in Study 5. In this study, between-person social relevance was negatively related to broadcast sharing intentions when adjusting for the other relevance variables in the model. In addition, this relationship did not differ significantly from zero in Studies 3 and 6. Together, this indicates that there is less consistency in the magnitude and direction of this relationship (compared to the other relevance variables) across studies. 
SHARING, SELF AND SOCIAL RELEVANCE

Table S8

Results from the combined/ adjusted multilevel models

\begin{tabular}{|c|c|c|c|c|c|}
\hline$\overline{\text { Model }}$ & Parameter & $\beta[95 \% \mathrm{CI}]$ & $d f$ & $t$ & $p$ \\
\hline \multirow[t]{4}{*}{ Study 1 broadcast } & Self between & $0.31[0.25,0.37]$ & 2097.70 & 10.85 & $<.001$ \\
\hline & Self within & $0.16[0.14,0.18]$ & 833.22 & 17.79 & $<.001$ \\
\hline & Social between & $0.19[0.13,0.24]$ & 2073.58 & 6.59 & $<.001$ \\
\hline & Social within & $0.14[0.13,0.16]$ & 781.23 & 16.49 & $<.001$ \\
\hline \multirow[t]{4}{*}{ Study 2 broadcast } & Self between & $0.23[0.12,0.33]$ & 548.60 & 4.23 & $<.001$ \\
\hline & Self within & $0.08[0.05,0.10]$ & 182.45 & 5.83 & $<.001$ \\
\hline & Social between & $0.24[0.13,0.34]$ & 538.39 & 4.45 & $<.001$ \\
\hline & Social within & $0.10[0.07,0.12]$ & 258.92 & 6.72 & $<.001$ \\
\hline \multirow[t]{4}{*}{ Study 3 broadcast } & Self between & $0.35[0.22,0.47]$ & 246.17 & 5.35 & $<.001$ \\
\hline & Self within & $0.13[0.08,0.18]$ & 115.16 & 4.86 & $<.001$ \\
\hline & Social between & $0.10[-0.03,0.23]$ & 242.43 & 1.54 & .130 \\
\hline & Social within & $0.09[0.05,0.14]$ & 128.47 & 3.88 & $<.001$ \\
\hline \multirow[t]{4}{*}{ Study 3 narrowcast } & Self between & $0.25[0.13,0.38]$ & 252.18 & 3.99 & $<.001$ \\
\hline & Self within & $0.11[0.06,0.16]$ & 91.050 & 4.28 & $<.001$ \\
\hline & Social between & $0.22[0.09,0.34]$ & 245.30 & 3.45 & $<.001$ \\
\hline & Social within & $0.16[0.11,0.21]$ & 109.51 & 6.18 & $<.001$ \\
\hline \multirow[t]{4}{*}{ Study 4 broadcast } & Self between & $0.42[0.34,0.49]$ & 134.43 & 11.03 & $<.001$ \\
\hline & Self within & $0.33[0.28,0.38]$ & 120.99 & 13.08 & $<.001$ \\
\hline & Social between & $0.15[0.07,0.22]$ & 136.50 & 3.83 & $<.001$ \\
\hline & Social within & $0.22[0.18,0.27]$ & 130.95 & 9.96 & $<.001$ \\
\hline \multirow[t]{4}{*}{ Study 5 broadcast } & Self between & $0.78[0.65,0.90]$ & 322.02 & 12.28 & $<.001$ \\
\hline & Self within & $0.21[0.18,0.24]$ & 220.10 & 13.76 & $<.001$ \\
\hline & Social between & $-0.12[-0.24,0.00]$ & 319.53 & 1.88 & .060 \\
\hline & Social within & $0.10[0.07,0.13]$ & 199.76 & 7.33 & $<.001$ \\
\hline \multirow[t]{4}{*}{ Study 5 narrowcast } & Self between & $0.57[0.43,0.70]$ & 316.41 & 8.42 & $<.001$ \\
\hline & Self within & $0.13[0.09,0.16]$ & 240.21 & 7.33 & $<.001$ \\
\hline & Social between & $0.07[-0.06,0.20]$ & 316.03 & 1.02 & .310 \\
\hline & Social within & $0.20[0.17,0.23]$ & 235.61 & 11.72 & $<.001$ \\
\hline \multirow[t]{4}{*}{ Study 6 broadcast } & Self between & $0.46[0.33,0.60]$ & 376.82 & 6.97 & $<.001$ \\
\hline & Self within & $0.17[0.14,0.19]$ & 316.63 & 12.71 & $<.001$ \\
\hline & Social between & $0.10[-0.03,0.23]$ & 374.09 & 1.46 & .140 \\
\hline & Social within & $0.14[0.12,0.16]$ & 250.99 & 11.81 & $<.001$ \\
\hline \multirow[t]{4}{*}{ Study 6 narrowcast } & Self between & $0.42[0.30,0.53]$ & 380.44 & 7.25 & $<.001$ \\
\hline & Self within & $0.11[0.08,0.14]$ & 279.68 & 7.92 & $<.001$ \\
\hline & Social between & $0.17[0.06,0.29]$ & 378.34 & 3.03 & $<.001$ \\
\hline & Social within & $0.26[0.23,0.29]$ & 281.19 & 17.57 & $<.001$ \\
\hline
\end{tabular}

Note. "Within" parameters refer to the person-centered level 1 predictors, whereas "between" parameters refer to grand-mean centered level 2 predictors. Coefficients are in standardized units. Degrees of freedom $(d f)$ were calculated using the Satterthwaite approximation.

Finally, for Studies 3, 5, and 6, we tested whether the relationships differed as a function of sharing type and directly compared broad- and narrowcast sharing intentions. Overall, the relationship between social relevance within- and between-person tended to be more strongly 
related to sharing intentions when narrowcasting than when broadcasting, whereas self-relevance tended to be more weakly related to sharing intentions when narrowcasting than when broadcasting (Table S9).

Table S9

Results from the sharing type interaction models

\begin{tabular}{|c|c|c|c|c|c|}
\hline Model & Parameter & $\beta[95 \% \mathrm{CI}]$ & $d f$ & $t$ & $p$ \\
\hline \multirow[t]{9}{*}{ Study 3} & Sharing type & $-0.00[-0.04,0.04]$ & 1987.51 & -0.08 & .940 \\
\hline & Social between & $0.11[-0.01,0.23]$ & 281.29 & 1.74 & .080 \\
\hline & Social within & $0.10[0.05,0.15]$ & 248.94 & 3.64 & $<.001$ \\
\hline & Self between & $0.34[0.22,0.46]$ & 284.52 & 5.60 & $<.001$ \\
\hline & Self within & $0.13[0.07,0.18]$ & 180.74 & 4.48 & $<.001$ \\
\hline & Social between $\mathrm{x}$ Sharing type & $0.10[0.03,0.16]$ & 1926.52 & 3.04 & $<.001$ \\
\hline & Social within $\mathrm{x}$ Sharing type & $0.05[-0.01,0.11]$ & 1927.26 & 1.54 & .120 \\
\hline & Self between $x$ Sharing type & $-0.08[-0.14,-0.01]$ & 1926.33 & -2.40 & .020 \\
\hline & Self within $x$ Sharing type & $-0.01[-0.07,0.05]$ & 1938.52 & -0.29 & .770 \\
\hline \multirow[t]{9}{*}{ Study 5} & Sharing type & $-0.00[-0.02,0.02]$ & 5741.47 & 0.00 & 1.000 \\
\hline & Social between & $-0.14[-0.27,-0.02]$ & 346.17 & -2.27 & .020 \\
\hline & Social within & $0.10[0.06,0.13]$ & 372.59 & 6.17 & $<.001$ \\
\hline & Self between & $0.81[0.68,0.93]$ & 347.20 & 12.78 & $<.001$ \\
\hline & Self within & $0.21[0.18,0.24]$ & 408.53 & 12.96 & $<.001$ \\
\hline & Social between $\mathrm{x}$ Sharing type & $0.23[0.18,0.28]$ & 5436.83 & 9.12 & $<.001$ \\
\hline & Social within $\mathrm{x}$ Sharing type & $0.11[0.08,0.14]$ & 5436.83 & 6.98 & $<.001$ \\
\hline & Self between $x$ Sharing type & $-0.26[-0.31,-0.21]$ & 5436.83 & -10.14 & $<.001$ \\
\hline & Self within $x$ Sharing type & $-0.09[-0.12,-0.06]$ & 5436.83 & -5.58 & $<.001$ \\
\hline \multirow[t]{9}{*}{ Study 6} & Sharing type & $-0.00[-0.02,0.02]$ & 7181.45 & 0.00 & 1.000 \\
\hline & Social between & $0.13[0.01,0.25]$ & 418.42 & 2.14 & .030 \\
\hline & Social within & $0.12[0.10,0.15]$ & 509.14 & 9.41 & $<.001$ \\
\hline & Self between & $0.45[0.33,0.57]$ & 419.87 & 7.39 & $<.001$ \\
\hline & Self within & $0.16[0.14,0.19]$ & 543.19 & 12.43 & $<.001$ \\
\hline & Social between $\mathrm{x}$ Sharing type & $0.04[-0.01,0.08]$ & 6801.62 & 1.46 & .140 \\
\hline & Social within $\mathrm{x}$ Sharing type & $0.14[0.11,0.17]$ & 6801.63 & 9.93 & $<.001$ \\
\hline & Self between $x$ Sharing type & $-0.02[-0.07,0.03]$ & 6801.62 & -0.92 & .360 \\
\hline & Self within $x$ Sharing type & $-0.06[-0.09,-0.03]$ & 6801.72 & -4.03 & $<.001$ \\
\hline
\end{tabular}

Note. "Within" parameters refer to the person-centered level 1 predictors, whereas "between" parameters refer to grand-mean centered level 2 predictors. The reference group for sharing type is broadcast sharing intentions. Coefficients are in standardized units. Degrees of freedom $(d f)$ were calculated using the Satterthwaite approximation.

\section{Preregistered mediation analyses including a single mediator}

We estimated four within-person mediation models (http://www.pagegould.com/r/indirectm/m/) testing the degree to which the effect of the experimental condition (self v. control, or social v. control) on sharing intentions was mediated by self-relevance in the self condition or social relevance in the social condition, estimating these models separately for broadcasting and narrowcasting. The raw units were retained here (versus standardizing) to 
facilitate interpretation in meaningful units. Bootstrapping was used to generate $95 \%$ confidence intervals.

For the self condition (Figure S8A), $81 \%$ of the total effect was mediated by changes in self-relevance for broadcast sharing intentions, and 79\% was mediated by changes in selfrelevance for narrowcast sharing intentions. A similar pattern was observed for the social condition (Figure S8B); 119\% of the total effect was mediated by changes in social relevance for broadcast sharing intentions, and $64 \%$ was mediated by changes in social relevance for narrowcast sharing intentions.
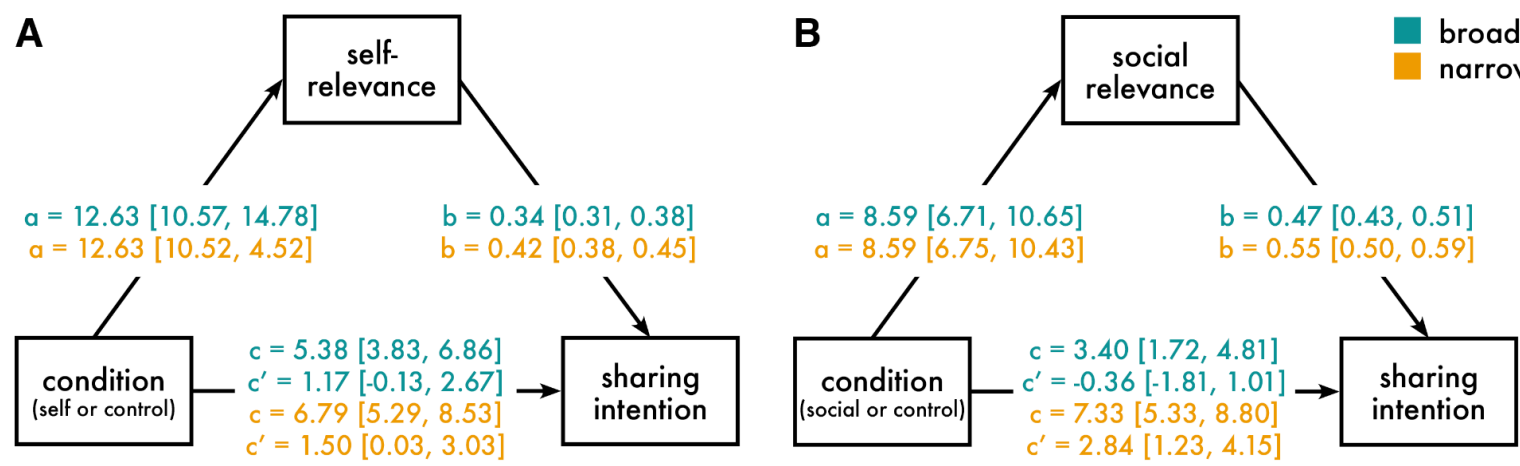

Figure S8. Path diagrams of the within-person multilevel mediation models for the (A) self condition and (B) social condition. Parameter estimates and bootstrapped $95 \%$ confidence intervals are reported for broadcast and narrowcast sharing intentions separately. $\mathrm{c}=$ total effect (direct + indirect effect of condition on sharing intention); $\mathrm{c}^{\prime}=$ direct effect.

\section{Post-hoc causal study analyses}

Moderation by article content type. We explored whether the effectiveness of the experimental manipulations was moderated by article content type (general health or climate change) in Study 6. To do so, we estimated the multilevel models reported in the main manuscript, but included content type and its interactions with experimental condition (all models) and sharing type (sharing intention model only). Compared to health content, climate content was rated as 1) more self-relevant, 2) less socially relevant, and 3) having lower sharing intentions when narrowcasting compared to broadcasting (Figure S9, Table S10). Finally, although there were mean level differences between content types, the effects of the experimental manipulation were similar across content; that is, content type did not moderate the effects of experimental condition on self-relevance, social relevance, or sharing intentions. 
SHARING, SELF AND SOCIAL RELEVANCE

Table S10

Results from the moderation analyses

\begin{tabular}{lcccc}
\hline Self-relevance & \multicolumn{5}{c}{} \\
\hline Parameter & $\beta[95 \% \mathrm{CI}]$ & $d f$ & $t$ & $p$ \\
\hline Control (intercept, health) & $51.33[48.66,54.00]$ & 386.91 & 37.77 & $<.001$ \\
Self & $13.30[10.27,16.34]$ & 234.52 & 8.64 & $<.001$ \\
Social & $4.88[1.75,8.02]$ & 224.15 & 3.07 & $<.001$ \\
Content (climate) & $3.30[0.51,6.10]$ & 396.79 & 2.32 & .020 \\
Self x Content (climate) & $-2.71[-6.26,0.84]$ & 253.60 & 1.50 & .130 \\
Social x Content (climate) & $0.61[-3.30,4.51]$ & 241.31 & 0.31 & .760 \\
& & & & \\
Social relevance & & & &
\end{tabular}

\begin{tabular}{lcccc}
\hline Parameter & $\beta[95 \% \mathrm{CI}]$ & $d f$ & $t$ & $p$ \\
\hline Control (intercept, health) & $61.45[59.02,63.88]$ & 387.62 & 49.71 & $<.001$ \\
Self & $7.72[5.22,10.22]$ & 239.9 & 6.09 & $<.001$ \\
Social & $7.22[4.27,10.18]$ & 230.84 & 4.82 & $<.001$ \\
Content (climate) & $-5.82[-8.26,-3.38]$ & 394.4 & 4.70 & $<.001$ \\
Self x Content (climate) & $1.33[-2.11,4.78]$ & 273.77 & 0.76 & .450 \\
Social x Content (climate) & $3.10[-0.35,6.55]$ & 236.9 & 1.77 & .080 \\
& & & & \\
Sharing intentions & & & & \\
\hline Parameter & $\beta[95 \% \mathrm{CI}]$ & $d f$ & $t$ & $p$ \\
Control (intercept, health, broadcasting) & $44.39[41.26,47.53]$ & 477.23 & 27.85 & $<.001$ \\
Self & $4.28[2.05,6.52]$ & 7084.00 & 3.76 & $<.001$ \\
Social & $3.94[1.70,6.17]$ & 7067.02 & 3.45 & $<.001$ \\
Sharing type & $2.09[0.08,4.11]$ & 1504.98 & 2.04 & .040 \\
Content (climate) & $1.45[-0.56,3.46]$ & 1695.61 & 1.41 & .160 \\
Self x Sharing type & $2.23[-0.78,5.24]$ & 7101.11 & 1.45 & .150 \\
Social x Sharing type & $3.98[0.97,6.99]$ & 7082.51 & 2.59 & .010 \\
Self x Content (climate) & $1.47[-1.60,4.55]$ & 7301.86 & 0.94 & .350 \\
Social x Content (climate) & $-1.38[-4.46,1.71]$ & 7291.96 & 0.87 & .380 \\
Sharing type x Content (climate) & $-3.91[-6.28,-1.54]$ & 6964.98 & 3.23 & $<.001$ \\
Self x Sharing type x Content (climate) & $-0.25[-4.40,3.89]$ & 7063.14 & 0.12 & .900 \\
Social x Sharing type x Content (climate) & $-0.76[-4.92,3.40]$ & 7053.97 & 0.36 & .720 \\
\hline
\end{tabular}

Note. The reference group for sharing type is broadcast sharing intentions, health for content type, and control for experimental condition. Degrees of freedom $(d f)$ were calculated using the Satterthwaite approximation. 

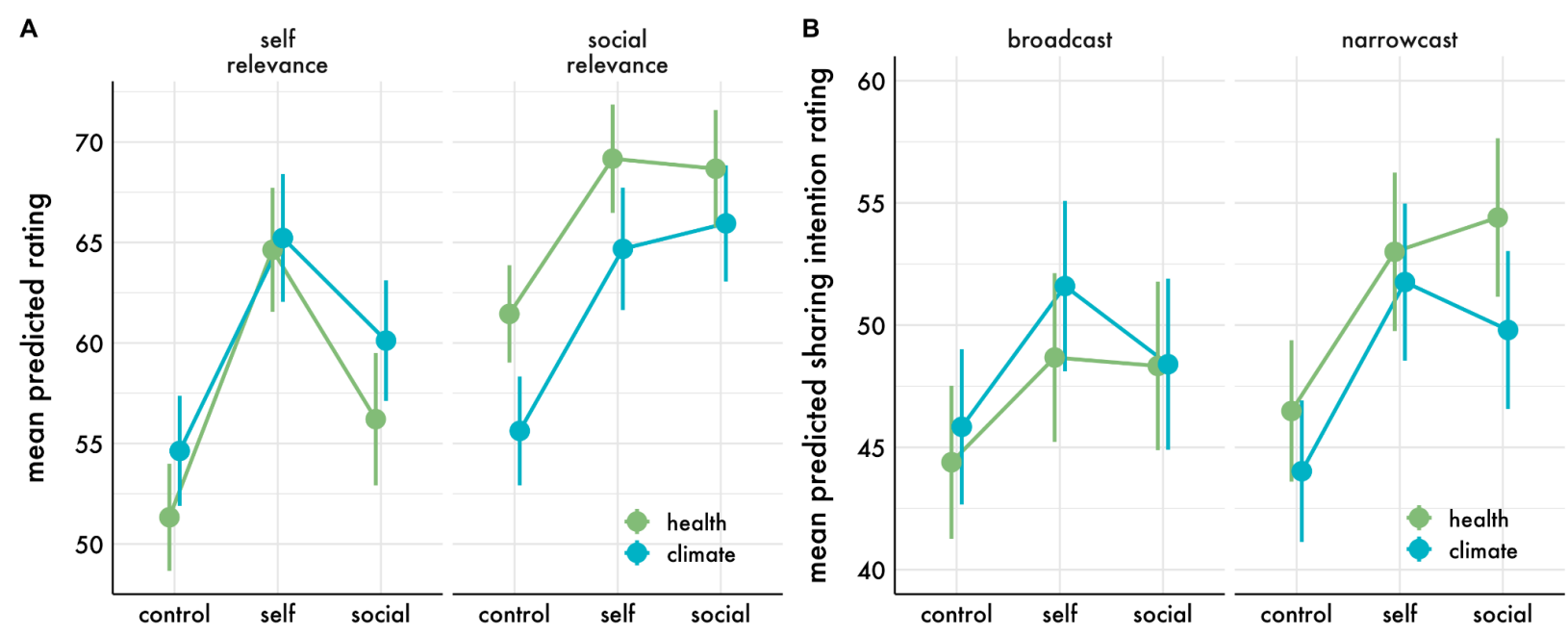

Figure S9. Mean predicted (A) self and social relevance ratings and (B) broad- and narrowcast sharing intentions as a function of experimental condition (self, social, or control) and article content type (health or climate). Error bars are $95 \%$ confidence intervals.

Depth of processing. To examine the potential role of depth of processing in the experiment, we conducted a post-hoc sensitivity analysis using word count as a proxy for depth of processing. Consistent with hypothesis that depth the experimental manipulations increased the depth of processing of the messages, participants wrote more in the self $(b=3.08,95 \% \mathrm{CI}$ $[2.60,3.56], t(3725.05)=12.63, p<.001)$ and social $(b=3.05,95 \%$ CI $[2.57,3.54], t(3727.22)$ $=12.43, p<.001)$ conditions compared to the control condition. Because word count was also positively related to self $(b=0.50,95 \%$ CI $[0.32,0.67], t(273.86)=5.62, p<.001)$ and social relevance $(b=0.48,95 \%$ CI $[0.33,0.63], t(236.60)=6.45, p<.001)$, we tested the degree to which the effects of the experimental manipulations on relevance was mediated by word count. For the self experimental manipulation, we found that $19 \%$ of the total effect on self-relevance and $13 \%$ of the total effect on social relevance was mediated by word count. For the social experimental manipulation, we found that $15 \%$ of the total effect on social relevance and $17 \%$ of the total effect on self-relevance was mediated by word count. This suggests that depth of processing may partially explain the effect of the experimental manipulations on self and social relevance, but is not sufficient to explain the full effect.

\section{References}

Bakdash, J. Z., \& Marusich, L. R. (2017). Repeated Measures Correlation. Frontiers in Psychology, 8, 456. https://doi.org/10.3389/fpsyg.2017.00456

Bates, D. \& Maechler, M. (2019). Matrix: Sparse and Dense Matrix Classes and Methods. R package version 1.2-18. https://CRAN.R-project.org/package=Matrix

Bates, D., Maechler, M., Bolker, B., \& Walker, S. (2015). Fitting Linear Mixed-Effects Models Using lme4. Journal of Statistical Software, 67(1), 1-48. https://doi.org/10.18637/jss.v067.i01. 
Bolker, B. \& Robinson, D. (2021). broom.mixed: Tidying Methods for Mixed Models. R package version 0.2.7. https://CRAN.R-project.org/package=broom.mixed

Bürkner, P-C. (2017). brms: An R Package for Bayesian Multilevel Models Using Stan. Journal of Statistical Software, 80(1), 1-28. doi:10.18637/jss.v080.i01

Canty, A., \& Ripley, B. (2019). boot: Bootstrap R (S-Plus) Functions. R package version 1.3-24.

Henry, L., \& Wickham, H. (2020). purrr: Functional Programming Tools. R package version 0.3.4. https://CRAN.R-project.org/package=purrr

Kassambara, A. (2020). ggpubr: 'ggplot2' Based Publication Ready Plots. R package version 0.4.0. https://CRAN.R-project.org/package=ggpubr

Kay, M. (2022). tidybayes: Tidy Data and Geoms for Bayesian Models. doi: 10.5281/zenodo.1308151

Kleiman, E. (2021). EMAtools: Data Management Tools for Real-Time Monitoring/Ecological Momentary Assessment Data. R package version 0.1.4. https:/CRAN.Rproject.org/package $=$ EMAtools

Kuznetsova, A., Brockhoff, P. B., \& Christensen, R. H. B. (2017). lmerTest Package:Tests in Linear Mixed Effects Models. Journal of Statistical Software, 82(13), 1-26. https://doi.org/10.18637/jss.v082.i13

Makowski, D., Ben-Shachar, M.S., Patil, I. \& Lüdecke, D. (2020). Automated Results Reporting as a Practical Tool to Improve Reproducibility and Methodological Best Practices Adoption. CRAN. Available from https://github.com/easystats/report

Masur, Philipp K. \& Scharkow, M. (2020). specr: Conducting and Visualizing Specification Curve Analyses. Available from https://CRAN.R-project.org/package=specr

Silge, J. \& Robinson, D. (2016). tidytext: Text Mining and Analysis Using Tidy Data Principles in R. Journal of Open Source Software, 1(3). doi: 10.21105/joss.00037

Vaughan, D. and Dancho, M. (2021). furrr: Apply Mapping Functions in Parallel using Futures. $\mathrm{R}$ package version 0.2.2. https://CRAN.R-project.org/package=furrr

Wickham, H. \& Hester, J. (2020). readr: Read Rectangular Text Data. R package version 1.4.0. https://CRAN.R-project.org/package=readr

Wickham, H. (2016) ggplot2: Elegant Graphics for Data Analysis. Springer-Verlag New York.

Wickham, H. (2019). stringr: Simple, Consistent Wrappers for Common String Operations. R package version 1.4.0. https://CRAN.R-project.org/package=stringr

Wickham, H. (2021). forcats: Tools for Working with Categorical Variables (Factors). R package version 0.5.1. https://CRAN.R-project.org/package=forcats

Wickham, H. (2021). tidyr: Tidy Messy Data. R package version 1.1.3. https://CRAN.Rproject.org/package $=$ tidyr

Wickham, H., Averick, M., Bryan, J., Chang, W., McGowan, L. D. A., François, R., ... \& Yutani, H. (2019). Welcome to the Tidyverse. Journal of open source software, 4(43), 1686. doi: https://doi.org/10.21105/joss.01686

Wickham, H., François, R., Henry, L., \& Müller, K. (2021). dplyr: A Grammar of Data Manipulation. R package version 1.0.7. https:/CRAN.R-project.org/package=dplyr 
Yihui Xie (2021). knitr: A General-Purpose Package for Dynamic Report Generation in R. R package version 1.31 .

Zhu, H. (2020). kableExtra: Construct Complex Table with 'kable' and Pipe Syntax. R package version 1.3.1. https://CRAN.R-project.org/package=kableExtra 